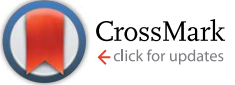

Cite this: RSC Adv., 2017, 7, 11462
Received 17th November 2016 Accepted 25th January 2017

DOI: 10.1039/c6ra26931a

rsc.li/rsc-advances

\section{Vascularization of LBL structured nanofibrous matrices with endothelial cells for tissue regeneration $\uparrow$}

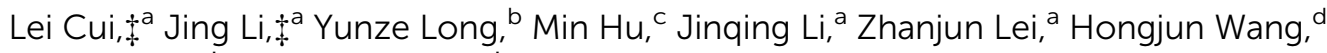 \\ Rong Huang ${ }^{\star a}$ and Xueyong $\mathrm{Li}^{\star a}$
}

To engineer functional vascular structures for reconstruction in tissue engineering, we evaluated the feasibility of layer-by-layer (LBL) isotropic and anisotropic structured poly( $\varepsilon$-caprolactone) (PCL)/ cellulose based nanofibers via electrospinning and LBL techniques in this study. The morphology of both fibers was analyzed using field emission scanning electron microscopy (FE-SEM) and atomic force microscopy (AFM). The aligned nanofibrous scaffold surface was nonthrombogenic as assessed using a platelet adhesion test, and the antithrombogenicity of modified nanofibrous mats was increased greatly with increased coating bilayers. Besides, human umbilical vein endothelial cells (HUVECs) were then seeded onto the LBL structured nanofiber meshes and analyzed for cell adhesion, proliferation and migration by FE-SEM, 3-(4,5-dimethylthiazol-2-yl)-2,5-diphenyltetrazolium bromide (MTT), cell tracking and cell migration assay. Moreover, the phenotypic expressions of HUVECs on LBL structured nanofibrous matrices with either isotropic or anisotropic fiber organizations were studied by immunofluorescent staining. Our data found that aligned nanofibers could guide morphogenesis and regulate cytoskeleton organization of HUVECs, and further promote in vitro prevascularization by facilitating phenotype-related protein expression and capillary-like tube formation as compared to randomly oriented nanofibers. Furthermore, the implantation in vivo of aligned composite scaffolds seeded with VECs demonstrated that the promoted host vessel infiltrated deep into the scaffolds and integrated with in vitro prefabricated vascular structures with increasing coating bilayers. Together, these findings supported our notion that the combination of aligned nanofibrous scaffolds and prevascularization could therefore serve as a promising strategy for the development of implantable functional vascular grafts by promoting rapid vascularization.

\section{Introduction}

To date, attempts to develop tissue engineering vascular grafts for replacing large-diameter (inner diameter $>6 \mathrm{~mm}$ ) blood vessels have been encouraging. However, the long-term patency of small diameter (inner diameter $<6 \mathrm{~mm}$ ) vascular grafts has remained disappointing over the past decade due to thrombus formation $^{\mathbf{1}}$ and intimal hyperplasia. ${ }^{2}$ From this point of view, tissue-engineered vessels consisting of vascular cells and novel

\footnotetext{
${ }^{a}$ Department of Plastic Surgery, Tangdu Hospital, Fourth Military Medical University, Xi'an 710038, Shaanxi, China. E-mail: lixueyong641123@163.com; 19881208huang@163.com; Fax:+862984777440; Tel: +862984777440

${ }^{b}$ College of Physics, Qingdao University, Qingdao 266071, China

${ }^{c}$ Department of Applied Chemistry, School of Science, Xi'an Jiaotong University, Xi'an 710049, Shaanxi, China

${ }^{d}$ Department of Chemistry, Chemical Biology and Biomedical Engineering, Stevens Institute of Technology, Hoboken, NJ 07030, USA

$\dagger$ Electronic supplementary information (ESI) available. See DOI: 10.1039/c6ra26931a

\$ Co-first author with the same contribution to this work.
}

supporting scaffolds ${ }^{3,4}$ may help to expedite endothelialization of the vessel lumen, which could lead to decreased thrombosis and neointimal thickening. Toward this end, a successful tissue engineered vascular graft should possess fundamental biological qualities including supporting cell growth and expansion and anticoagulant activity until the endothelial cell lining is fully achieved (hemocompatibility). ${ }^{5}$

Recently, prevascularization with endothelial cells have been applied to promote vascularization of implanted scaffolds in vivo. ${ }^{6,7}$ In this system, the vascular cells, such as endothelial cells, are pre-seeded onto the scaffolds and incubated to form vasculature $^{8}$ in vitro and improve the patency of implanted small-diameter grafts. $^{9-11}$ Moreover, cell seeded vascular construct facilitate graft maturation and promote remodeling when implanted in vivo. ${ }^{\mathbf{1 1}}$ Hence both the viable endothelial cells and supporting scaffold play a major role in achieving patent engineered grafts. ${ }^{12}$ Therefore, it is important to construct artificial microenvironment equipped with nonthrombogenic interface to serve as cell carriers and provide structural support for aiding the attachment, proliferation and 
phenotypic maintenance of endothelial cells ${ }^{13-15}$ and further promote vascularization upon implantation.

Among the strategies for promoting vascularization, the electrospinning technology has enabled the creation of fibers on the micro- and nano-scale with enormous potential with similar structure of native extracellular matrix (ECM), suitable mechanical properties of blood vessels ${ }^{\mathbf{1 6}}$ and controlled degradation during remodeling ${ }^{\mathbf{1 7 - 1 9}}$ in vascular tissue engineering. Besides, the high surface area to volume ratio could allow for the direct attachment of ECM ligands, growth factors, and other biomolecules onto fiber surfaces to locally modulate cell and tissue function..$^{\mathbf{2 0 - 2 4}}$ Apart from the substrate topology, the chemical composition of the biomaterials also provides signaling cues to cells. In particular, the type I collagen, known as the most abundant matricellular protein in the arterial ECM network, provides the primary structural framework of the blood vessel wall and harbors signaling cues for vascular cells. ${ }^{25}$ To better mimic the native ECM blood vessels, electrospun collagen fibers have been fabricated, ${ }^{26-28}$ however, the rapid degradation and poor mechanical properties when untreated with cross-linking lead to its unstability and limit its further clinical application. Electrospinning a blend solution of cellulose acetate (CA) and polycaprolactone (PCL), a biocompatible and biodegradable synthetic polyester, can yield rather stable nanofibers and support the adhesion, proliferation and migration of skin fibroblasts as previously described.$^{29}$ To immobilize the above ECM proteins onto nanofibers, functionalization of nanofibers is performed by layer-by-layer (LBL) self-assembly technique firstly reported by Decher ${ }^{30}$ because LBL modification has been proven to be very effective strategy to promote the biological function of skin cells. Besides, to improve the antithrombogenicity of the small-diameter vascular grafts, a natural polymer, chitosan (CS) was chosen as the other assembled material since the positive charge of CS retarded the blood coagulation, which was consistent with the results of the thrombin generation test as reported by He et al. ${ }^{31}$ In addition, it has been noted that the orientation of nanofibers are able to support cell adhesion and guide cellular behavior, ${ }^{32}$ e.g., aligned collagen I fibrillar matrices were proved to provide essential cell-matrix interaction and guide corneal endothelial cell alignment along the fibrils. ${ }^{33}$ Though the exact mechanism remains unclear, it is believed that the electrospun nanofiber could regulate cellular phenotype, which is critical to determine the functionality of tissue-engineered vascular grafts in vitro and subsequently influence the quality of vascularization upon in vivo grafting.

Taken together, we proposed the hypothesis that mechanically appropriate and nonthrombogenic scaffolds for application as vascularization for transplantation and even off-theshelf available vascular grafts could be produced by combining LBL modified scaffold designs and prevascularization with endothelial cells. ${ }^{34}$ Our study is turning toward the tissue engineering approach, which utilizes both biomimetic nanofibrous microstructure with isotropic (fibers collected randomly with no alignment) and anisotropic (fibers collected with alignment) topology and prevascularization to promote the function of vascular grafts.
To verify this hypothesis, in this context, isotropic (fibers collected randomly with no alignment) and anisotropic (fibers collected with alignment) PCL-cellulose fibers were fabricated by electrospinning with a blend of PCL and CA and then biofunctionalized by alkaline hydrolysis and the following deposition of CS and type I collagen in this work. The morphology and structure of prepared isotropic and anisotropic nanofibers were firstly studied using emission scanning electron microscope (FE-SEM) and atomic force microscopy (AFM) measurements. Secondly, the biological effect of anisotropic nanofibrous scaffolds on antithrombogenicity, VECs morphology, function, and capillary-like structure formation in vitro were characterized. More importantly, the implantation in vivo of prepared electrospun scaffold and Masson trichrome staining were applied to explore whether the aligned composite nanofibrous scaffold seeded with VECs could anastomose with host vessels and accelerate vascular infiltration throughout the whole tissue construct.

\section{Results and discussion}

\subsection{Fabrication and characterization of the LBL structured nanofiber meshes}

As for tissue-engineered grafts, rapid vascularization is considered to be a crucial issue especially for cell-containing tissue constructs in regenerative medicine,$^{34}$ the lack of vascularization and oxygen in the center could lead to ischemia followed by necrosis after implantation in vivo. ${ }^{35}$ Thus how to improve rapid vascularization and more rapid integration with native tissues remains a very important and challenging issue. On this condition, various strategies including biomaterial modifications and VECs seeding have been investigated to facilitate rapid vascularization through larger tissue constructs. ${ }^{7}$

In this paper, on the basis of our previous study on the LBL modified film-coated nanofibrous matrices which could modulate the adhesion and proliferation of $\mathrm{NHFs},{ }^{29}$ we adopted a parametric approach of designing nanofibrous scaffold with different orientation and then functionalized the surface of electrospun polycaprolactone (PCL)-cellulose nanofibers with CS and type I collagen via LBL technique to enhance the attachment and proliferation of HUVECs.

The morphology and diameter distribution of modified nanofibers were examined by FE-SEM (Fig. 1A-E). Non-woven PCL-cellulose nanofibers were significantly aligned with porous structure, which is desirable in ECM-mimicking materials. The as-spun nanofibrous and LBL structured (CS/COL $)_{n}$ films coated scaffolds showed similar surface morphologies which consisted of continuous and oriented nanofibers with diameters ranging from 100 to $700 \mathrm{~nm}$. The SEM micrographs appeared that the fabricated matrices had a solid surface with interconnected voids among the fibers, presenting a porous network. The distribution of the fiber diameters showed that all scaffolds had approximately $90 \%$ of the nanofibers in $100-$ $400 \mathrm{~nm}$ diameter range (Fig. 1F). Besides, the diameter of unmodified, LBL structured (CS/COL) $)_{n}$ films coated mats was $221 \pm 70 \mathrm{~nm}(n=0), 224 \pm 94 \mathrm{~nm}(n=5), 230 \pm 65 \mathrm{~nm}(n=10)$, $288 \pm 107 \mathrm{~nm}(n=15)$ and $292 \pm 109 \mathrm{~nm}(n=20)$, respectively 

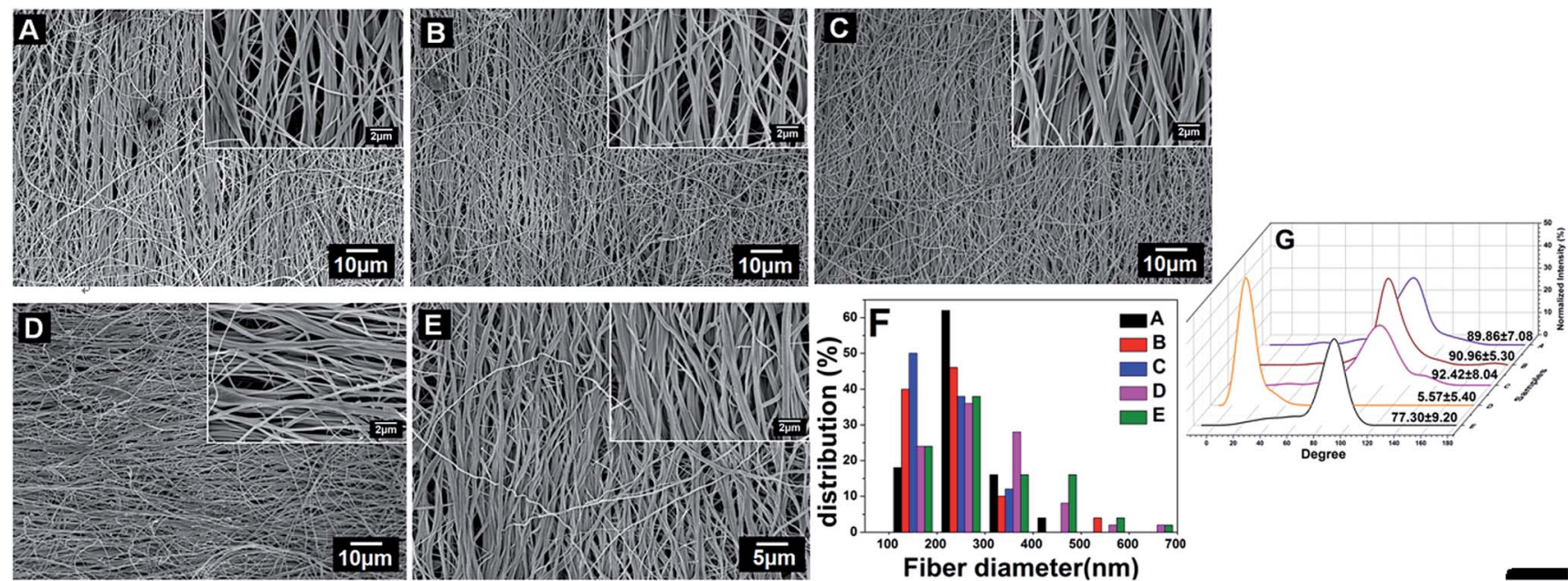

Fig. 1 FE-SEM images of (A) electrospun aligned PCL/cellulose template and LBL structured nanofibrous mats assembled by $(C S / C O L)_{n}$ : $(B) n=$ 5, (C) $n=10$, (D) $n=15$, (E) $n=20$. (F) Histogram showing diameter distribution of nanofibers based on SEM images. (G) Normalized intensity plots against the angle of acquisition for nanofibrous matrices.

while $n$ varied from 5 to 20 (Table 1 ). Hence the diameter difference among the prepared LBL structured mats was not statistically significant with the deposition of CS and collagen. To quantitatively analyze the fiber alignment, FFT analysis was performed on the SEM images of randomly selected area $(n=5)$ to characterize the anisotropy and assign a numerical value to fiber orientation. The narrow peak of the normalized intensity values in prepared nanofibrous matrices revealed that all the PCL/cellulose nanofibers and LBL structured mats aligned along a single axis (Fig. 1G).

It is noteworthy that the fiber diameter increased from $358 \pm$ $118 \mathrm{~nm}$ to $541 \pm 197 \mathrm{~nm}$ before and after CS and collagen were alternatively assembled on the randomly oriented PCL-cellulose template for 20 coating bilayers as described in our previous work. ${ }^{29}$ Of note, the space between fibers did not narrow after coating bilayers, which was different from the morphology of isotropic nanofibers with the same parameter. The reason might be the orientation of fibers. Briefly, more junctions, bigger protuberances as well as denser bundles formed between bundles of fibers were obtained in the resultant isotropic nanofibrous mats with unaligned structure with increasing coating bilayers (data not shown), leading to larger fiber diameter than that of anisotropic fibers.

Surface roughness of nanofibers has been identified to be an important factor that may influence cell attachment, proliferation and migration. ${ }^{36,37} \mathrm{Xu}$ et al. found that the endothelial cell

Table 1 The average fiber diameter of prepared nanofibrous mats

\begin{tabular}{ll}
\hline Samples & $\begin{array}{l}\text { Average } \\
\text { diameter }(\mathrm{nm})\end{array}$ \\
\hline PCL/cellulose & $221 \pm 70$ \\
$(\mathrm{CS} / \mathrm{COL})_{5}$ & $224 \pm 94$ \\
$(\mathrm{CS} / \mathrm{COL})_{10}$ & $230 \pm 65$ \\
$(\mathrm{CS} / \mathrm{COL})_{15}$ & $288 \pm 107$ \\
$(\mathrm{CS} / \mathrm{COL})_{20}$ & $292 \pm 109$
\end{tabular}

function was enhanced on the smooth solvent-cast surface rather than on the rough electrospun surface of poly(L-lactic acid). ${ }^{38}$ Therefore Bruker atomic force microscope (AFM) was utilized to determine the surface roughness of individual nanofibers. The morphology of aligned and randomly oriented PCL-cellulose mats (Fig. $2 \mathrm{~A}$ and $\left.\mathrm{A}^{\prime}\right)$ and $(\mathrm{CS} / \mathrm{COL})_{20}$ film-coated nanofibers (Fig. 2B and $\mathrm{B}^{\prime}$ ) was examined. From the analysis of AFM, it is obviously observed that the surface of aligned nanofibers showed a rather smoother surface than the randomly oriented nanofibers, a lot of undulations were observed on the surface of the latter. In order to better characterize the roughness of these matrices, parameters $\left(R_{\mathrm{q}} / R_{\mathrm{rms}}\right.$, root mean square roughness) were determined by using Bruker Nanoscope Analysis software supplied with AFM.

As shown in Fig. 2A and B, the roughness of isotropic PCLcellulose mats alone was $223.52 \pm 67.72 \mathrm{~nm}$ (Fig. 2A), while it increased to $338.69 \pm 11.78 \mathrm{~nm}$ after coating with CS and collagen bilayers $(p<0.001)$ (Fig. 2B), and the same trend was observed between them for the anisotropic specimens $76.31 \pm$ $8.09 \mathrm{~nm}$ for PCL-cellulose mats and $119.42 \pm 6.97 \mathrm{~nm}$ for (CS/ $\mathrm{COL})_{20}$ films coated mats $(p<0.001)$ (Fig. $2 \mathrm{~A}^{\prime}$ and $\left.\mathrm{B}^{\prime}\right)$, which elucidated that the fiber alignment seems to induce a slight decrease in the surface roughness while the LBL modification led to a slight increase, which might result from the difference in the fiber diameter and fiber alignment between LBL structured isotropic and anisotropic nanofibers with the same coating bilayers. Briefly, both the anisotropic and isotropic fiber diameter increased with $(\mathrm{CS} / \mathrm{COL})_{20}$ film-coating as measured by FE-SEM images, which was consistent with the work reported by Milleret et al., he analyzed poly(lactic-co-glycolic acid) (PLGA) or polyester urethane (PEU) fibers from the nanoscale to approximately 7 microns and found that fiber surface roughness increased with increasing fiber diameter. ${ }^{39}$ Besides, the slight decrease in the surface roughness of the aligned samples might be ascribed to the reduced release of residual stresses imposed by the processing during the solvent evaporation. 


\subsection{Platelet adhesion examination}

Progression of late thrombosis remains as the major limitation of currently available vascular grafts. The adsorption of blood proteins initiated blood response when a exogenous biomaterial contact with blood, followed by platelet adhesion and the activation of coagulation pathways, thus resulting in thrombus formation. ${ }^{40}$ Since the main orientation of this study is to promote endothelialization on the surface of LBL structured nanofibrous matrices, it is vital to evaluate the platelet attachment onto scaffold because the attachment of platelets at the implant site in vivo has been associated with thrombosis and subsequent restenosis. More importantly, to fabricate a successful vascular construct, the scaffold must ensure low platelet activation while maintaining the platelet's role in hemostasis and angiogenesis. ${ }^{\mathbf{4 1}}$

To examine whether the electrospun scaffold could resist platelet adhesion, the isotropic and anisotropic nanofibrous mats with various coating bilayers were exposed to platelets under static conditions at $2 \mathrm{~h}$. It was previously described that the morphological criteria of activated platelets could be divided into five categories (dendritic, dendritic spread, spread, fully spread, and nonviable $)^{42}$ which is applied to assess the activation state of the platelets adhered to the surface of our prepared mats. Fig. 3 shows the CLSM images of platelet adhered to collagen I-coated stainless steel surface and electrospun anisotropic composite nanofibers. We found that both large quantities of platelet adhesion and activation are observed
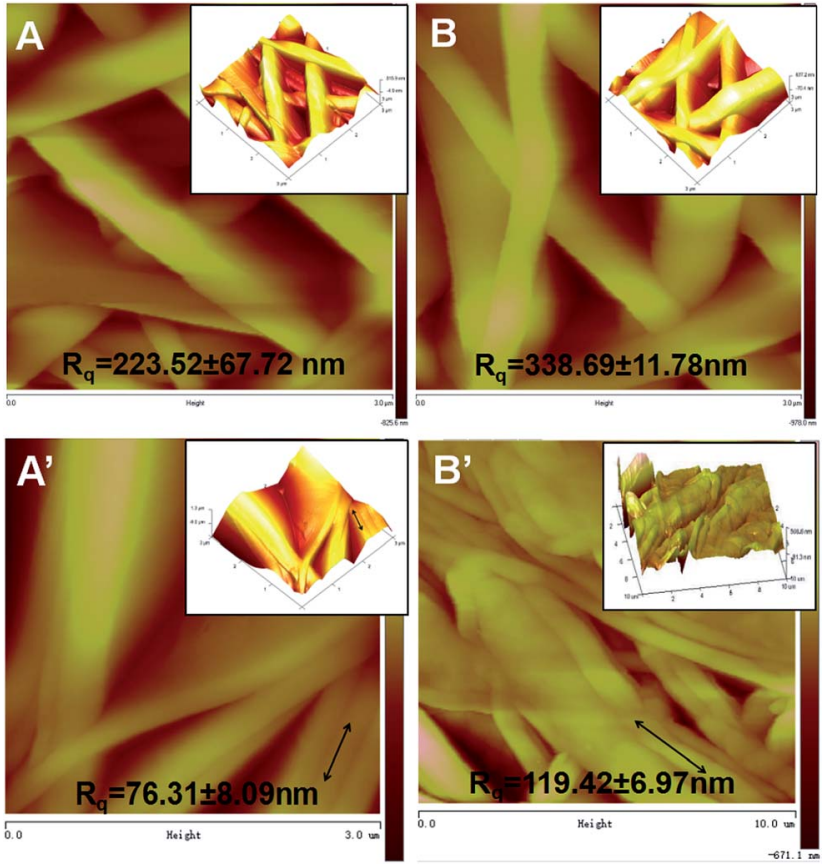

Fig. 2 Represented AFM images and the $R_{\mathrm{q}}$ (root mean square roughness) of randomly oriented ( $A$ and $B$ ) and aligned $\left(A^{\prime}\right.$ and $\left.B^{\prime}\right)$ nanofibrous mats observed at height mode: ( $A$ and $\left.A^{\prime}\right) P C L-c e l l u l o s e$ nanofibrous mats, ( $B$ and $B^{\prime}$ ) nanofibrous mats assembled by (CS/ $\mathrm{COL})_{20}$ coating bilayers. Solid line in the insets showed the direction of the fibers. on the pure collagen I-coated stainless steel surface (Fig. 3A). The number of platelets adhered on it was found to be $117 \pm 23$, while the prepared nanofibrous scaffolds resisted the adherence of these blood elements (Fig. 3B-E). The adhesion of platelets on PCL-cellulose nanofibers was $42 \pm 12$, showing that adhesion of platelets on pure collagen-coated surface was more than 2 folds higher than as-spun nanofibrous mats. With the CS/COL modification on the surface of as-spun PCL-cellulose mats, the mesh exhibited good blood compatibility and low blood coagulation. There was noticeable trend in the direction of decrease in platelet adhesion and absence of activation while increasing coating CS/COL bilayers on LBL modified mats, few cells can be found in the crevice of the fibers, and they retained a discoid shape which is similar to the original shape of the platelet at unactivated state. In contrast, the isotropic nanofibers possessed a flattened morphology with the presence of platelet pseudopodia to the underlying nanofibers, had relative high platelet deposition (see in ESI, Fig. S1 $\dagger$ ). More importantly, we observed that the LBL structured anisotropic $(\mathrm{CS} / \mathrm{COL})_{20}$ films coated nanofibrous mats presented a promising feature in terms of relatively low platelet adhesion $(7 \pm 2)$.

Since changes in surface chemical composition and roughness of sample surface both affect thrombogenicity, ${ }^{42}$ at least two reasons were responsible for this phenomenon. Firstly, the decrease in the platelet adhesion on the $(\mathrm{CS} / \mathrm{COL})_{n}$ films coated mats might be influenced by the surface chemistry, where hydrophilic repulsion occurs and prevented the direct contact between the platelets with the composite surface. He et al. found that the positive charge of CS retards blood thrombin generation and blood coagulation on CS films which is in contrast to prevailing views. The CS acted as a double-edged sword, on one hand, it promoted erythrocyte adhesion, fibrinogen adsorption, and platelet adhesion and activation, but it inhibited the activation of the contact system on the contrary, and the positive charge could not significantly promote the activation of nonadherent platelets in the bulk phase during the early stage of coagulation. ${ }^{31}$ Secondly, surface roughness can increase platelet adhesion and the presence of platelet pseudopodia to the underlying substrate. Thus there is an increase in platelet adhesion, spreading, and subsequent platelet activation..$^{39}$ Based on the AFM data, the lower surface roughness of anisotropic nanofibers resulted in less platelet adhesion and activation as confirmed by CLSM images, suggesting that prepared anisotropic LBL structured nanofibrous composite scaffold could be a potential candidate for anti-thrombogenicity, which is particularly useful for artificial blood prostheses.

\subsection{Cell adhesion and proliferation}

To monitor cell adhesion and viability on different substrates, the cell metabolic activity for the evaluation of survival and growth characteristics of HUVECs was determined by MTT assay. Fig. 4A summarized the cell viability on various substrates with different coating bilayers along with $24 \mathrm{~h}$ and $120 \mathrm{~h}$ incubation. The cell culture plate (TCPS) was used as the control group, it showed that HUVECs cultured in all nanofibrous scaffolds exhibited a similar growth pattern of time- 

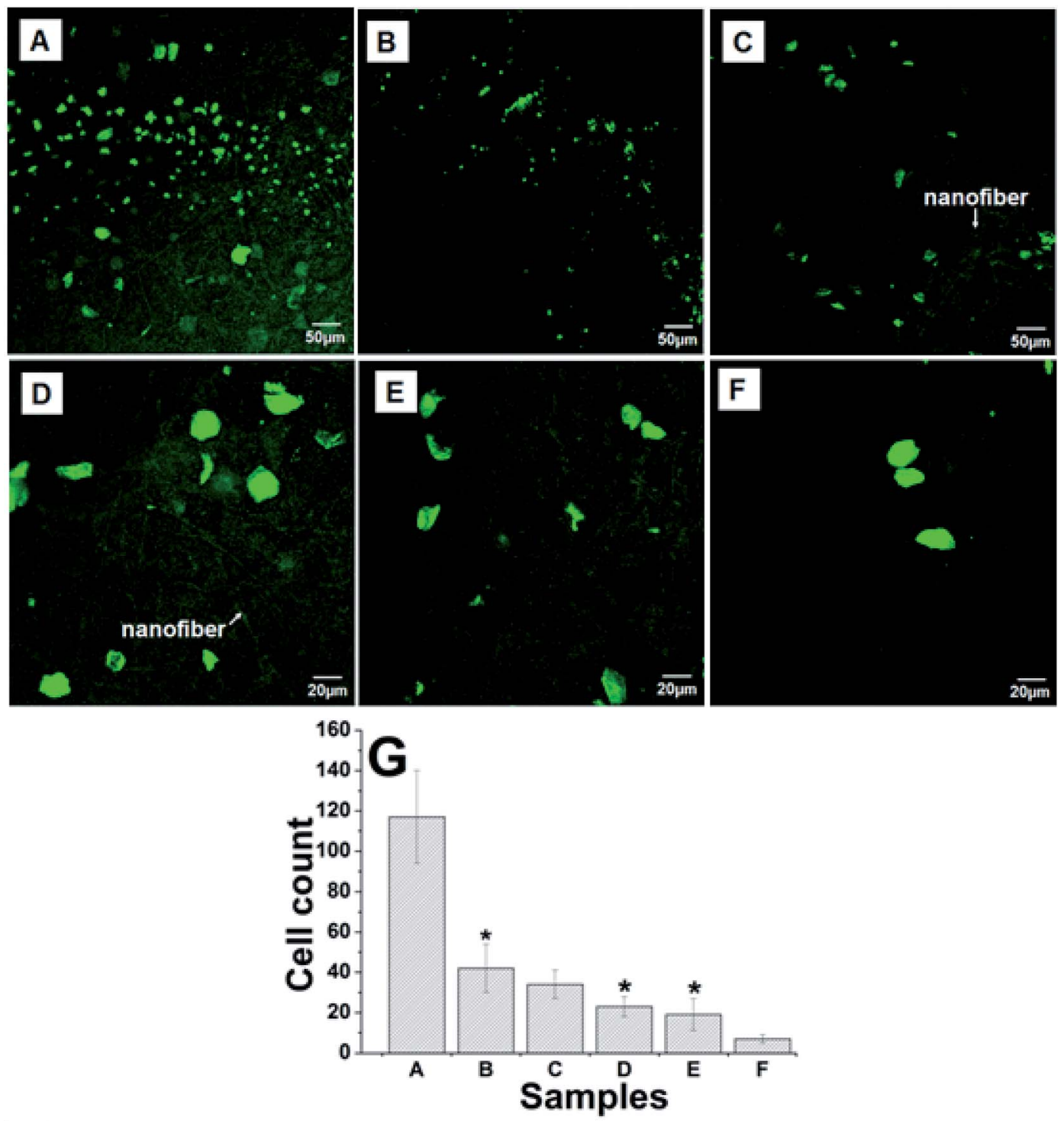

Fig. 3 The platelet adhesion on prepared (A) type I collagen coated surface, (B) PCL-cellulose mesh and nanofibrous mats coated by (CS/COL) : (C) $(\mathrm{CS} / \mathrm{COL})_{5}$, (D) $(\mathrm{CS} / \mathrm{COL})_{10}$; (E) $(\mathrm{CS} / \mathrm{COL})_{15}$; (F) $(\mathrm{CS} / \mathrm{COL})_{20}$. (G) The quantitative calculation of adherent blood platelets.

dependent increase of cell number during the culture period. The cell viability of HUVECs on PCL, cellulose and PCL-cellulose template evidenced the better biocompatibility with PCLcellulose than that of neat PCL or cellulose. Besides, compared with the cell viability of HUVECs on PCL-cellulose template, the addition of CS and collagen on PCL-cellulose template during LBL process led to higher cell viability. On day 5 after incubation, lower optical density was observed on TCPs than that on PCL-cellulose and (CS/COL) ${ }_{n}$, which was due to the $2 \mathrm{D}$ surface suitable for the monolayer. Although the overall cell number increased in all scaffolds during the culture period, the LBL structured films coatings modified by biocompatible CS and collagen would be more beneficial for cell migration and penetration, as well as further growth. Of note, the cell density on LBL structured mats coated with more coating bilayers was significantly higher at all time points. Hence it can be concluded that the number of coating bilayers played a critical role in the cell growth on nanofibrous matrices. Together, the MTT results indicated that the prepared nanofibrous mats promoted the survival of HUVECs during in vitro incubation.

Based on the MTT results, cell tracking method was also applied to monitor the effect of prepared isotropic and anisotropic $(\mathrm{CS} / \mathrm{COL})_{20}$ film-coated mats (Fig. 4B-E) on the cell viability, HUVECs were labeled with red CellTracker ${ }^{\mathrm{TM}}$ dye prior to seeding in vitro and then the constructs were visualized using CLSM. It was obvious that the viable cells which were positively stained red adhered on aligned $(\mathrm{CS} / \mathrm{COL})_{20}$ film-coated mats exhibited enhanced survival of HUVECs than that of the randomly oriented fibers, which provided evidence of the better biocompatibility of the aligned LBL modified nanofibrous scaffold, HUVECs successfully adhered to the surface within $24 \mathrm{~h}$ and the coverage continued to expand thereafter, leading 

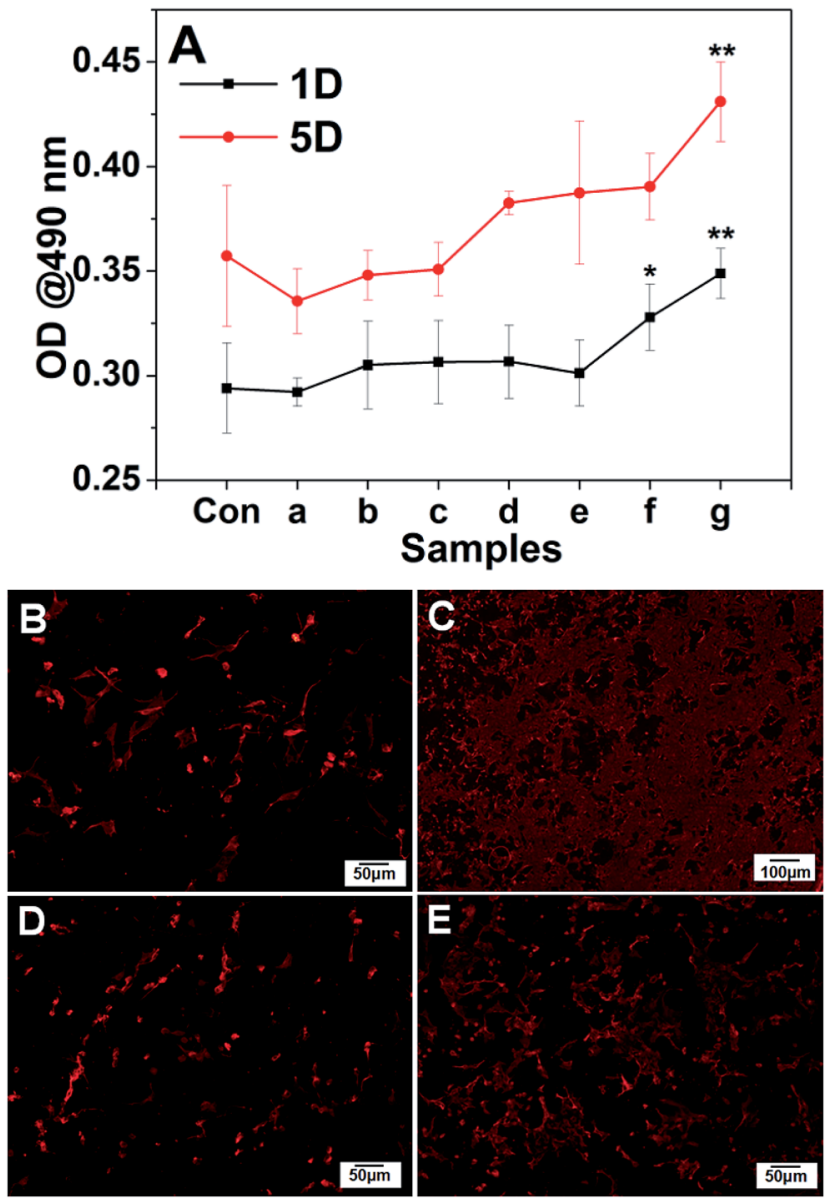

Fig. 4 (A) MTT assay for the cell viability of HUVECs incubated after 24 and $120 \mathrm{~h}$ alone (control group) and with aligned nanofibrous mats: (a) $\mathrm{PCL}$ nanofibrous matrix, (b) cellulose matrix, (c) PCL/cellulose template and LBL coated mats assembled by $(\mathrm{CS} / \mathrm{COL})_{n}:(\mathrm{d}) n=5$; (e) $n$ =10; (f) $n=15$; (g) $n=20$. Significant difference (vs. control, untreated group): $* p<0.05 ; * *<0.01$. (B-E) CellTrackerTM Red CMPTX labelled HUVECs co-cultured with (B and $C$ ) aligned (CS/COL) 20 LBL structured mats for $1 \mathrm{~d}(\mathrm{~B})$ and $5 \mathrm{~d}(\mathrm{C})$ and co-cultured with ( $\mathrm{D}$ and $\mathrm{E})$ randomly oriented $(\mathrm{CS} / \mathrm{COL})_{20} \mathrm{LBL}$ structured mats for $1 \mathrm{~d}(\mathrm{D})$ and $5 \mathrm{~d}(\mathrm{E})$.

to a cellular layer by day 5 , which was demonstrated by the efficient endothelialization of graft surfaces.

\subsection{Cell migration}

In order to evaluate the effects of LBL structured $(\mathrm{CS} / \mathrm{COL})_{20}$ nanofibrous matrices with aligned and randomly oriented morphology on HUVECs migration, an in vitro wound healing assay was performed by culturing cells on various matrices with wound gaps with an insert of $900 \mu \mathrm{m}$ (Fig. 5). The culture stained with crystal violet after $0 \mathrm{~d}, 3 \mathrm{~d}$ and $5 \mathrm{~d}$ revealed that the migration of HUVECs was significantly regulated by the underlying nanofibers with different orientation. HUVECs cocultured with isotropic fibers barely migrated into the wound gap after $3 \mathrm{~d}$ culture with gap distance of $725.6 \pm 28.7 \mu \mathrm{m}$ and only provided moderate wound coverage (about $66.3 \pm 16.5 \mu \mathrm{m}$ ) after $5 \mathrm{~d}$ (Fig. 5A). In contrast, on the LBL films coating matrices with obvious orientation, cells migration into the wound area was greatly enhanced after 3 d culture with gap distance of 639.8 $\pm 15.8 \mu \mathrm{m}$. The wound gap was completely closed when cells were migrated on the (CS/COL $)_{20}$ by $5 \mathrm{~d}$ (Fig. 5B), suggesting that the aligned nanofibrous scaffold with LBL modification modulated VECs activity by inducing significantly greater cellular outgrowth in vitro than non-patterned scaffolds. These results revealed that the promotion of HUVECs migration was induced by the collagen-containing nanofibrous matrices with aligned structure. Though the potential mechanisms of aligned nanofiber-mediated endothelial migration remained unclear, Nakayama et al. found that EC-seeded aligned nanofibrillar scaffolds promoted 3D cellular outgrowth in vitro by activating integrin $\alpha 1 .^{28}$

Prevascularization with seeded endothelial cells possess advantage of rapid host vessel anastomoses with preconstructed networks. ${ }^{43}$ However, the survival and function of endothelial cells can not be ensured due to inadequate oxygen and nutrient delivery. ${ }^{44}$ Our present study demonstrated the anisotropic LBL modified fibers with various aligned $(\mathrm{CS} / \mathrm{COL})_{n}$ films coatings displayed promoted adhesion, proliferation, spreading and migrating of HUVECs in vitro than that of isotropic fibers.

\subsection{Cell morphology and cytoskeleton organization}

To determine whether the direction of prepared viable nanofibrous mats could affect the cell orientation and polarity for vascular tissue engineering applications, we then assessed cellular morphology and spreading on the electrospun constructs after $72 \mathrm{~h}$ of incubation via scanning electron microscopy (SEM) images of the cells on the LBL structured (CS/ $\mathrm{COL})_{n}$ films coated scaffold surfaces (Fig. S2 $\dagger$ and 6). As observed in Fig. S1 (see in ESI $\dagger$ ), all nanofibrous matrices supported the attachment and growth of HUVECs similarly, while showing different cell morphology with distinct variation in filopodia- and lamellipodia-like extensions. In Fig. S2A, $\uparrow$ the cell maintained 3D structure and adhered well on the surface of (CS/ $\mathrm{COL})_{5}$ films coating nanofibrous network. Cells were shown to be spindle-shaped and distributed on the substrate surface, after pretreatment with $(\mathrm{CS} / \mathrm{COL})_{n}$ films coatings, the pronounced biocompatibility with HUVECs were observed. As shown in Fig. S2B-F, $\dagger$ when the coating bilayers increased from 5.5 to 15.5 , the cells were located at the subsurface of the nanofibers, even immigrated below several fibrous layers of fibers, and it exhibited that fibers crossed upon the cells and cells grew within the fibrous scaffold (Fig. S2G-H†). Moreover, several cells interacted with adjacent cells with forming numerous pseudopodia between them, which was characteristic of the promoted cell adhesion and spreading. The data demonstrated that the cell adhesion on LBL structured mats was significantly enhanced with increasing LBL coating bilayers, which was attributed to the adsorption/immobilization of serum proteins on deposited bilayer.

However, in comparison with the unidirectional fibers, the development of matrix-induced alignment of the VECs is depicted in the images in Fig. 6, the HUVECs attached and stretched on the nanofibers, displaying a flattened morphology 

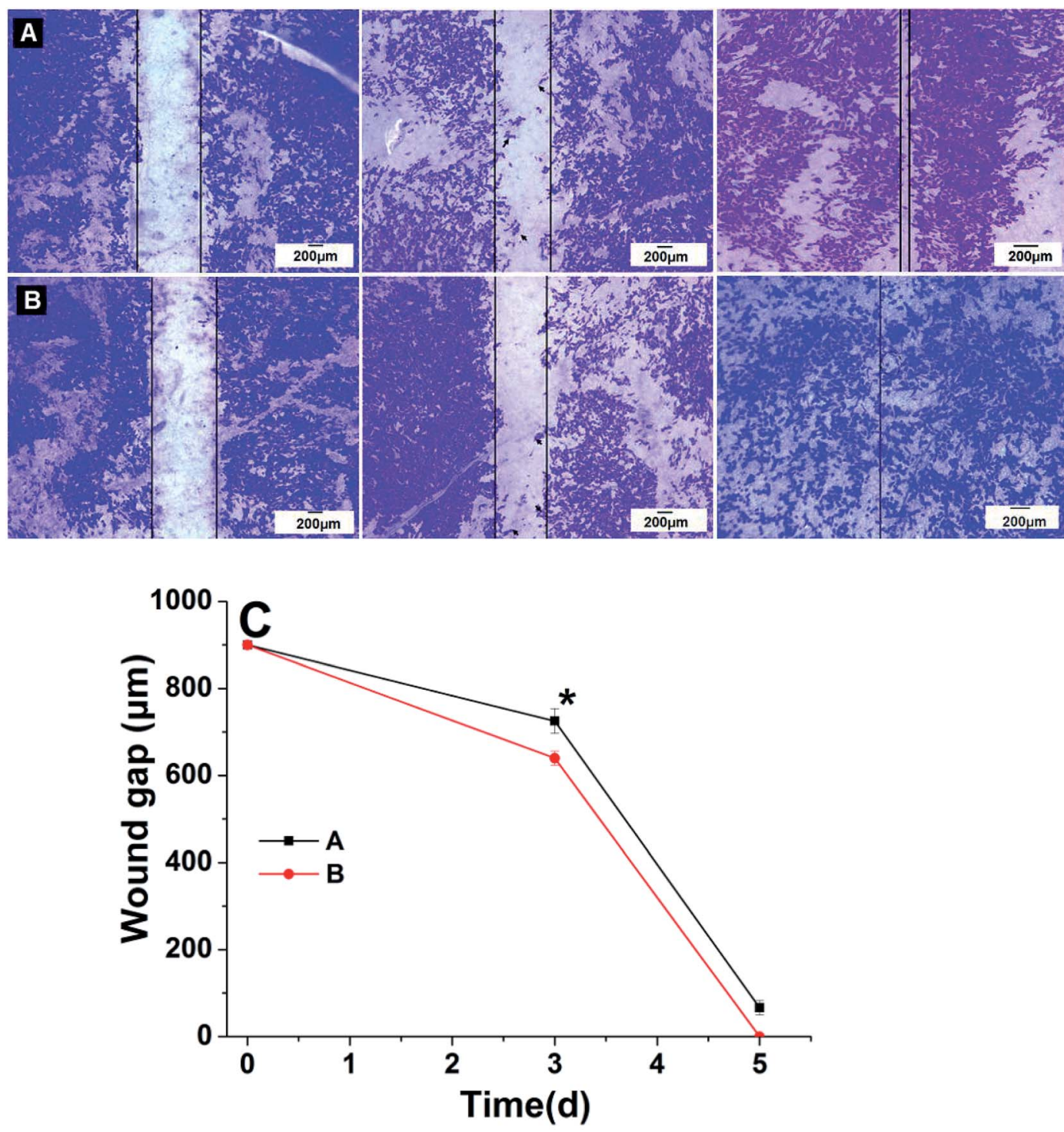

Fig. 5 Migration of HUVECs on nanofibrous matrices in wound healing assay in vitro. Cells were seeded on (A) aligned and (B) randomly oriented nanofibrous mats coated by (CS-collagen) 20 with an insert in the middle. After $24 \mathrm{~h}$, the insert was removed to generate a $0.9 \mathrm{~mm}$ "wound gap". Cells were allowed to migrate into the wound gap, and visualized after 0, 3 and 5 days. (C) Quantification of the "wound gap" distance between the front lines of migrating cells.

with numerous attachment sites with the underlying fibers, forming numerous attachment sites with the underlying fibers (Fig. 6A and B). The cell was intensively attached to the surfaces by forming long, numerous microvilli and extending lamellipodia, filopodia, and the microvilli tended to grow along the orientation of nanofibers when coating bilayers increased from 10 to 20.5 (Fig. 6C-F). The cells seeded onto aligned nanofibers responded to the anisotropy of the underlying surface by demonstrating a distinct elongated and polarized morphology along the fiber direction (Fig. 6G and $\mathrm{H}$ ).

We further investigated whether unaligned and aligned nanofibers could guide morphogenesis of HUVECs and regulate cytoskeleton organization. The F-actin cytoskeleton was visualized with TRITC-labeled phalloidin (Fig. 7). After 3 day culture, F-actin staining results exhibited that HUVECs displayed polygonal morphology on the isotropic matrices without orientation despite certain oriented actin filaments within individual cells (Fig. 7A). In contrast, the HUVECs on anisotropic matrices became elongated and exhibited numerous well-stretched actin bundles with obvious orientation along the direction of nanofibers (Fig. 7B). Moreover, closer examination of the actin organization showed compact bundles instead of individual actin filaments like those on isotropic matrices, which confirmed that the aligned LBL films coated nanofibers with CS and collagen deposition generally facilitated the cell adhesion process of HUVECs on nanofibers. In Nien's work, he fabricated poly( $\varepsilon$-caprolactone)/poly(ethylene oxide)/chitosan (CS) fibers in both aligned and random structures to investigate cell affinity. The results showed that the aligned PCL/PEO/chitosan ultrafine fibrous mat had the 

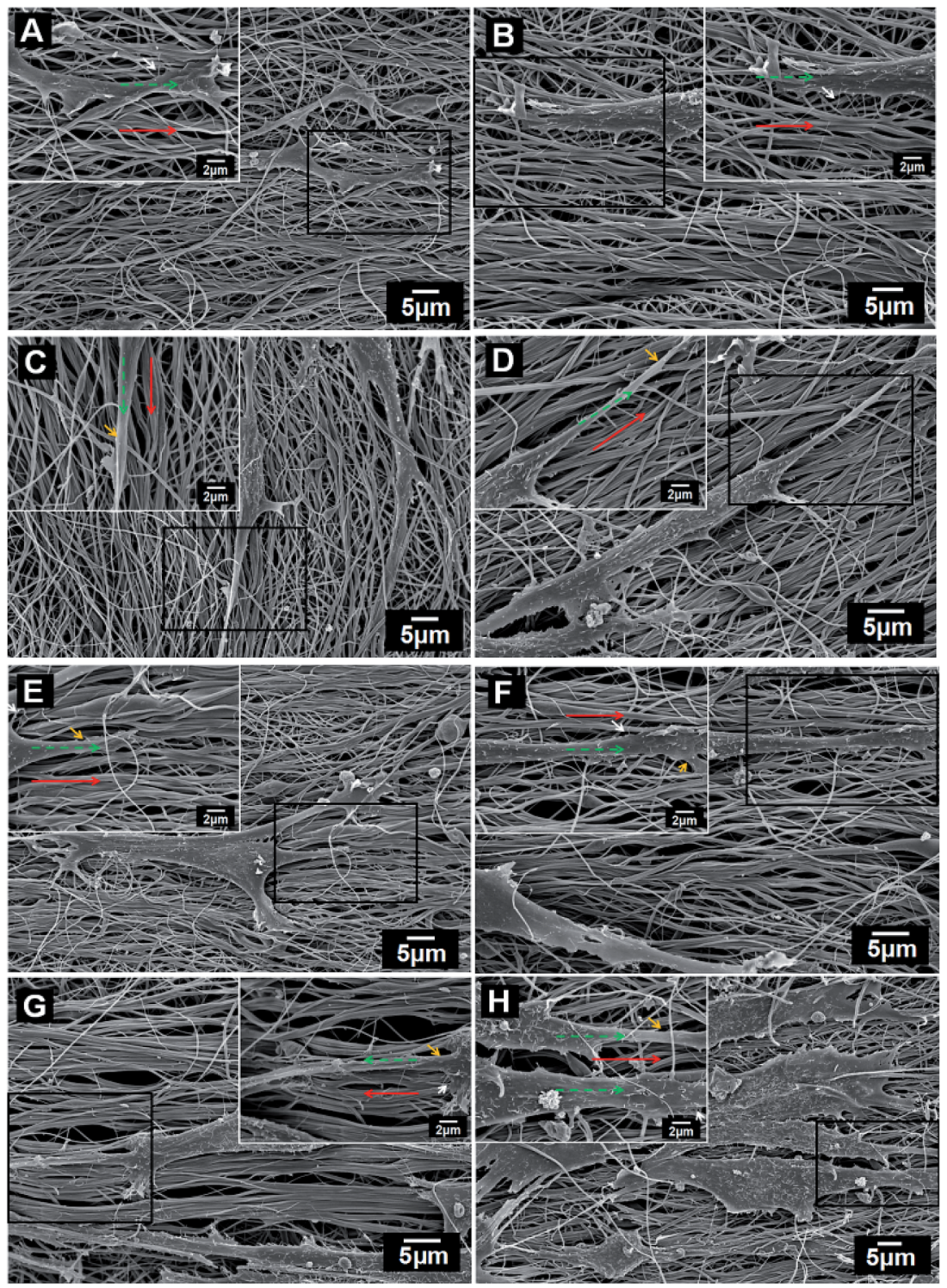

Fig. 6 Morphological observation by SEM. HUVECs in the presence with aligned nanofibrous mats coated by $(C S / C O L)_{n}$ after $72 \mathrm{~h}$ incubation: (A) $(\mathrm{CS} / \mathrm{COL})_{5}$, (B) $(\mathrm{CS} / \mathrm{COL})_{5.5}$, (C) $(\mathrm{CS} / \mathrm{COL})_{10}$, (D) $(\mathrm{CS} / \mathrm{COL})_{10.5}$, (E) $(\mathrm{CS} / \mathrm{COL})_{15}$, (F) $(\mathrm{CS} / \mathrm{COL})_{15.5},(\mathrm{G})(\mathrm{CS} / \mathrm{COL})_{20}$, (H) $(\mathrm{CS} / \mathrm{COL})_{20.5}$. Scale bars of the images and were $10 \mu \mathrm{m}$ and $5 \mu \mathrm{m}$, respectively. Red solid and green dashed line in the insets showed the direction of the fibers and cells while yellow and white arrow represented lamellipodia and filopodia, respectively.

capacity to induce cellular alignment and enhance cellular elongation. ${ }^{45}$ Collectively, the distribution and organization of cytoskeleton protein inside VECs were parallel to the direction of the nanofibers. Hence it seemed reasonable to arrive at the conclusion that the orientation of as-spun nanofibers and the following LBL modification played curial role in regulating the phenotypic alteration especially cell affinity of HUVECs.

In order to further elaborate whether the orientation of nanofibers affected the spatial distribution of focal adhesion plaques on cell membranes to study the functional development of HUVECs on the nanofibers during in vitro culture. The expression of focal adhesion marker with cell growth on both substrates is also shown in Fig. 8. It was showed that the vinculin evenly distributed across the cell membrane on both matrices. Interestingly, the vinculin of cells co-cultured with LBL modified isotropic PCL/COL films coated nanofibrous matrices was well expressed and it was located mostly at cellto-cell interfaces and cell membrane without forming tubelike structure at various time points (1, 3, 5, 7 and $14 \mathrm{~d}$ ). However, on anisotropic matrices, long segments of vinculin were stained along the nanofiber alignment (Fig. $8 \mathrm{~A}^{\prime}$ ), but on the isotropic matrices no preferred orientation was observed (Fig. 8A), and obvious tube-like structure via the cell-scaffold interaction was observed on aligned nanofibrous mats (Fig. $8 \mathrm{~B}^{\prime}$ and $\mathrm{E}^{\prime}$ ), which was of great benefit to blood vessel regeneration. 

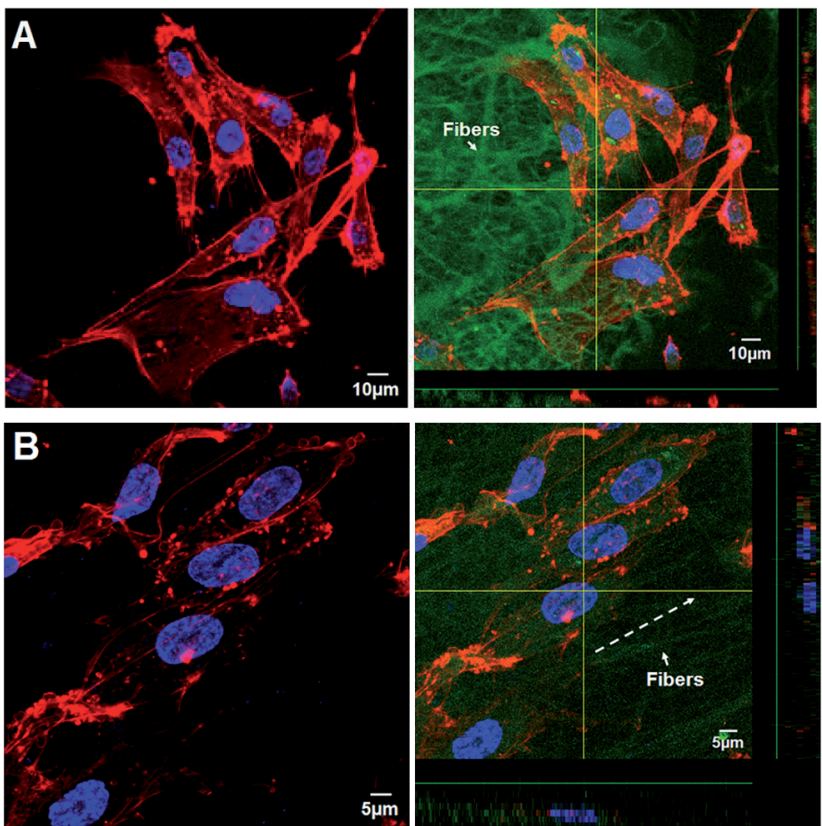

Fig. 7 Cytoskeleton development of HUVECs grown on the (A) randomly oriented and $(B)$ aligned $(\mathrm{CS} / \mathrm{COL})_{20}$ electrospun meshes. The nanofibers, F-actin, and nuclei were stained with FI-TC (green), TRITC-phalloidin (red) and DAPI (blue), respectively. The wellstretched actin bundles were visible when the cells were grown on both matrices. HUVECs successfully maintained their native morphology and formed filamentous actin-based stress fibers after $72 \mathrm{~h}$ incubation.

\subsection{Immunofluorescent staining}

Endothelial cell adhesion molecule CD31 was assessed by immunohistochemistry to observe vascularization in cells cocultured with prepared isotropic and anisotropic $(\mathrm{CS} / \mathrm{COL})_{20}$ films coated nanofibrous mats for each group (Fig. 9). A small, dotted pattern of CD31 stained red was clearly visible and well distributed within the cytoplasm and at intercellular junctions when HUVECs were grown on both matrices. The results suggested that cells on the aligned and unaligned nanofibers exhibited positive CD31-staining and kept typical VEC phenotype. However, the intensities of the fluorescence of the cells on randomly oriented scaffolds appeared to be less than those on aligned scaffolds at the same time point. Interestingly, positive expression of CD31 on aligned film-coated PCL-cellulose nanofibers indicated adequate inter endothelial contacts between adjacent cells with forming tube-like microstructure. The results indicated that the vascular cells seeded on aligned scaffolds synthesized more characteristic protein than those seeded on randomly oriented scaffolds. It could be known that the released CS and collagen had bioactivity to promote spreading and proliferating of HUVECs on the nanofibrous mats, which could synergically stimulate the proliferation and spreading of VECs.

According to previous literature, angiogenesis occurs when endothelial cells start to proliferate and to sprout from preexisting vessels and thereby forming new vessels, then the newly formed endothelial tubes finally mature by assembling a basement membranes (BMs) and recruiting smooth muscle cells or pericytes. The BMs plays an important role in angiogenesis (blood vessel formation). It is a dynamic, self assembled layer of proteins, glycoproteins, and proteoglycans formed by enveloping endothelial cells and pericytes of blood vessels. ${ }^{46}$ Among the above components, nidogen/entactin and heparan sulfate proteoglycan perlecan are well recognized as major and ubiquitous basement membrane components. ${ }^{47}$ Indeed, nidogen/ entactin, an invariant component of basement membranes, is a multifunctional protein containing binding sites which acts as a link between the extracellular matrix molecules including laminin 1, collagen IV and perlecan, and thereby participates in the assembly of BMs. ${ }^{48}$ Besides, perlecan, a heparan sulfate proteoglycan assembled into the vascular BMs, binds to a variety of cell surface and basement membrane proteins including integrins, laminin, and nidogen, and is required for the reconstitution of basement membrane-like structures in vitro. ${ }^{49}$ Erika Gustafsson et al. found that perlecan maintains microvessel integrity, mechanical stabilizing ${ }^{50}$ in vivo and modulates their formation in vitro. ${ }^{51}$

Given the important roles of nidogen and perlecan produced by the endothelial cells in vascular basement membrane (BM) formation and regeneration, ${ }^{47,51}$ nidogen- 2 and perlecan protein
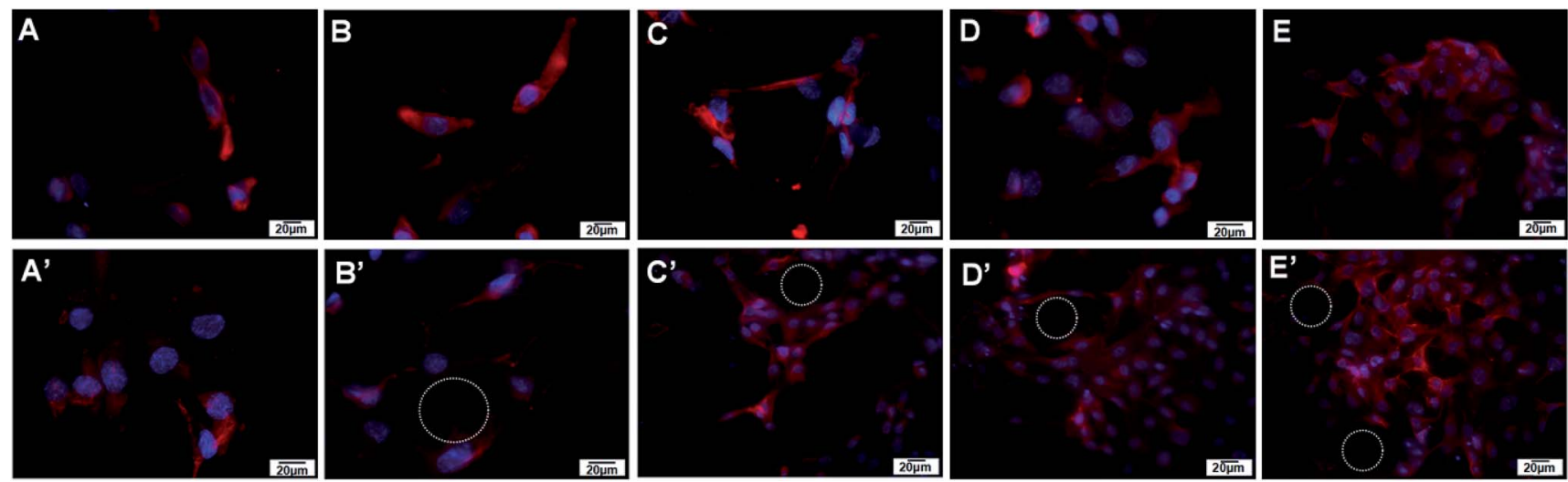

Fig. 8 Immunofluorescent staining of vinculin (green) in HUVECs on isotropic ( $A-E)$ and anisotropic ( $A^{\prime}$ and $\left.E^{\prime}\right) L B L$ structured nanofibrous matrices seeded for $1 d\left(A\right.$ and $\left.A^{\prime}\right), 3 d\left(B\right.$ and $\left.B^{\prime}\right), 5 d\left(C\right.$ and $\left.C^{\prime}\right), 7 d\left(D\right.$ and $\left.D^{\prime}\right)$ and $14 d\left(E\right.$ and $\left.E^{\prime}\right)$, respectively. Nuclei was stained with DAPI (blue). 


\section{Isotropic}
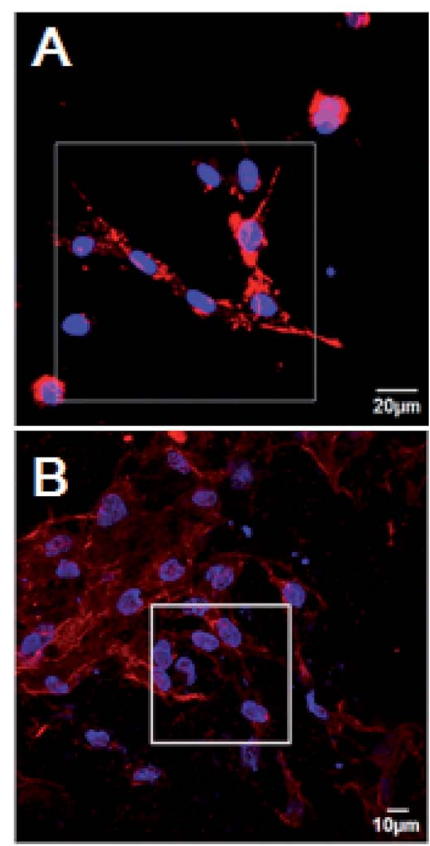

\section{(cose}

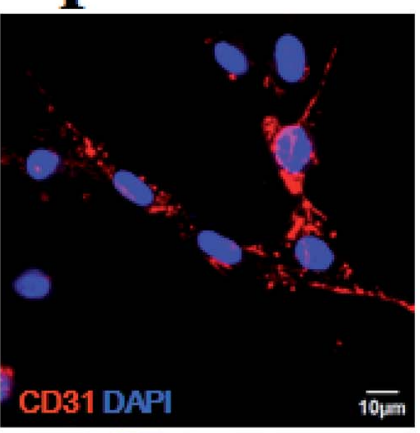

$10 \overline{\mathrm{p} m}$

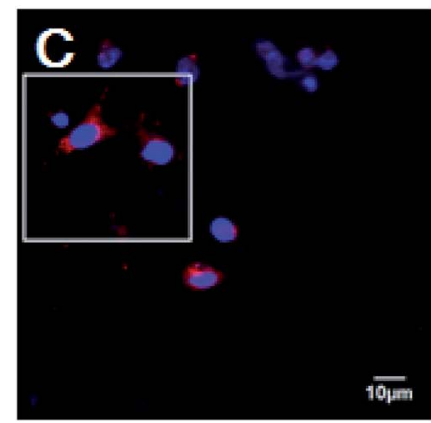

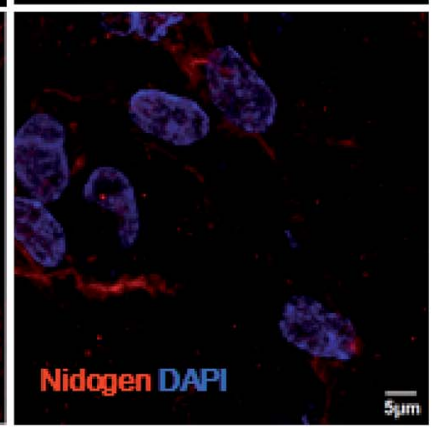

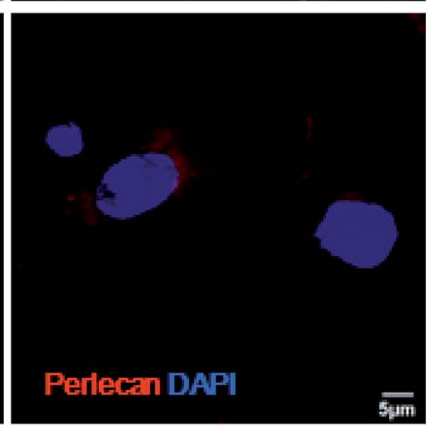

Anisotropic
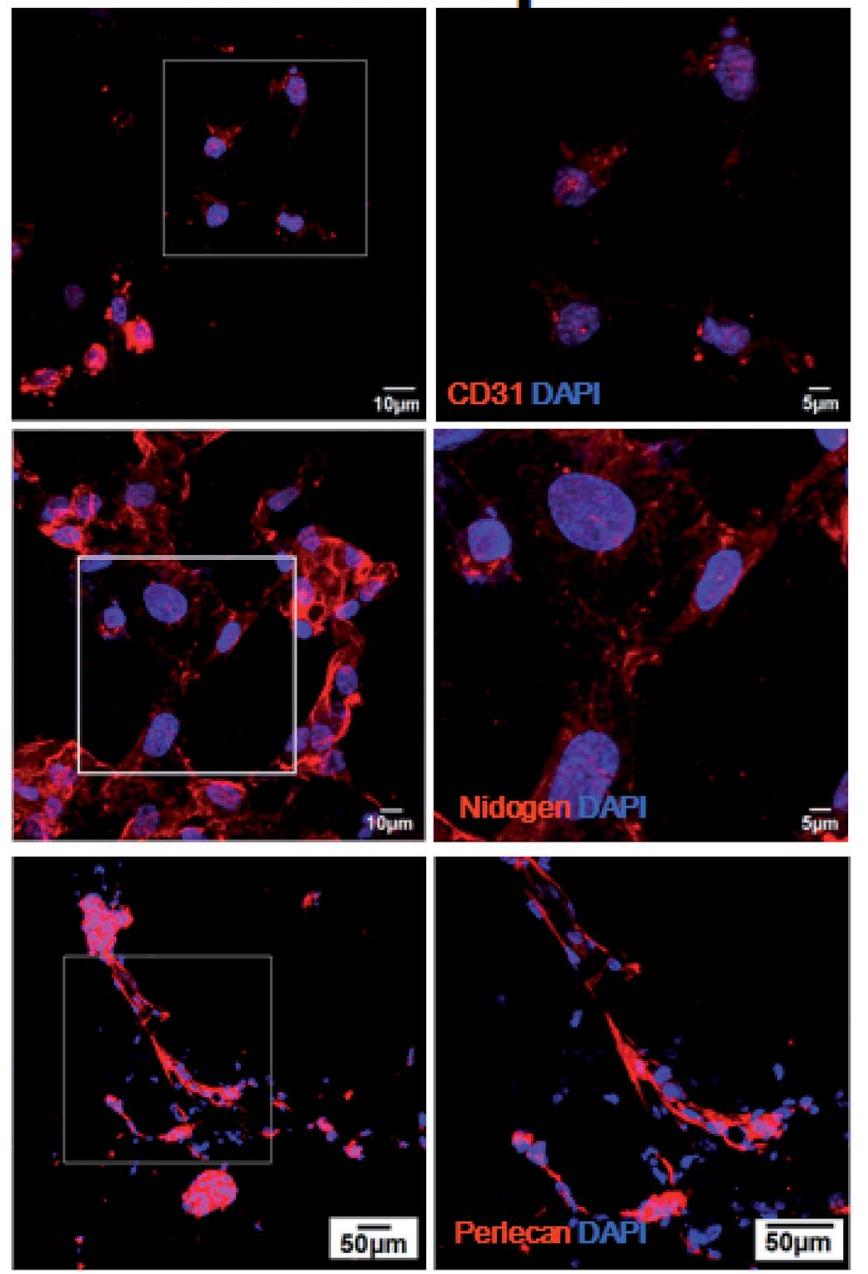

Fig. 9 Expression of CD31, nidogen and perlecan on HUVECs grown on randomly oriented and aligned $(\mathrm{CS} / \mathrm{COL})_{20} \mathrm{LBL}$ structured electrospun meshes. Cells positively expressed the representative endothelial cell markers (CD31) and basement membranes (BMs) protein (nidogen and perlecan).

expression was monitored with immunocytochemistry (Fig. 9). The synthesis of basement membrane (BM) protein nidogen (entactin) and perlecan possessed a similar trend as the expression level of CD31. Immunohistochemical staining for both perlecan and nidogen- 2 revealed a sharp and continuous reactivity in the VECs co-cultured with anisotropic $(\mathrm{CS} / \mathrm{COL})_{20}$ films coating nanofibers, faint immunoreactivity was detected in the cells for both BM components in isotropic (CS/COL $)_{20}$ films coated nanofibers. On unaligned nanofibers, nidogen was expressed but its location was restricted to single cells that have less inter-cellular adhesion. Nidogen-2 localization and expression was different in cells co-cultured with isotropic and anisotropic nanofibers, the expression of nidogen- 2 protein (red) was seen to be highest in the perinuclear organelle for cells on isotropic nanofibers whereas the expression was more diffuse and formed a mesh-like network throughout the cells on anisotropic nanofibers, and perlecan (red) was highly localized in cells on anisotropic nanofibers what appeared to be intracellular organelles whereas in the cells co-cultured with isotropic nanofibers the perlecan was seen more diffused throughout the cell body with forming tube-like microstructure. These results show that the levels of expression and localization of expression of nidogen-2 and perlecan are different in the cells co-cultured with nanofibers with different topological structure. These results indicate that HUVECs interact well with LBL structured film-coated PCL-cellulose nanofibers and the aligned nanofibers could significantly promote the prevascularization in vitro.

On the whole, when aligned nanofibers were compared to randomly oriented nanofibers, HUVECs showed stronger attachment, proliferation, higher expression of phenotyperelated proteins together with organized endothelial morphology in the assembly of focal adhesion proteins and cytoskeleton. These data demonstrated that the LBL aligned modified nanofibrous mats provided a combined function of promoting nutrient delivery, cell infiltration and distribution as well as cell proliferation to enhance in vitro scaffold prevascularization with HUVECs, therefore the aligned (CS/COL) $n$ 
films coated LBL structured nanofibrous scaffold were selected to perform the following in vivo implantation experiment.

\subsection{Anisotropic nanofibers and pre-seeded VECs additively promote rapid vascularization in vivo}

The schematic diagram of the in vivo implantation of prepared aligned nanofibrous meshes is displayed in Fig. S3 (see ESI†), to understand the mechanism by which the VEC-seeded aligned nanofibrous scaffolds induce angiogenesis, histological analysis of extracted tissue sections after 14 and 28 days was performed. Masson's trichrome stained sections showed the degradation behavior, collagen deposition and vascularization. At 2 week (Fig. S4 $\dagger$ ), all the scaffolds were well tolerated by the host animals, and no abnormal conditions were observed. The implanted as-spun PCL-cellulose mats did not appear to be heavily enveloped by fibrous tissues in most cases, they were able to retain their structural integrity and almost no signs of degradation were observed (Fig. S4A $\dagger$ ). Although most of these materials still held their structural integrity, signs of degradation were observable on the $(\mathrm{CS} / \mathrm{COL})_{5},(\mathrm{CS} / \mathrm{COL})_{10}$ and $(\mathrm{CS} /$ $\mathrm{COL})_{15}$ films coatings which were stained blue with visible fiber fragmentations (Fig. S4B-Di). As a part of tissue ingrowth, few vessels were detected in $(\mathrm{CS} / \mathrm{COL})_{20}$ films coatings (Fig. S4E†).

However, the aligned nanofibrous mats that were grafted into SD rat subcutaneous tissue almost degraded within 28 days (Fig. 10), and no inflammation could be seen on the nanofiber surfaces or in the surrounding tissues (except in the early stage wound). The scaffolds could not be observed obviously and could hardly hold their structural integrity after implantation. The subcutaneously implanted $(\mathrm{CS} / \mathrm{COL})_{n}$ films coated mats were filled with layers of collagen fiber bundles and thin fibrous layer. Besides, the degradation rate of LBL structures mats were significantly enhanced with increasing coating bilayers as evidenced by gradually reduced nanofibrous mats remained at implantation site. Moreover, the implanted materials were almost completely degraded, and only few fiber fragmentations distributed in the loose connective tissue at the implantation site. The level of regenerated microvessels were significantly promoted after implanting $(\mathrm{CS} / \mathrm{COL})_{n}$ films coatings with larger coating bilayers.

Based on the insight gained, the implanted fibers were degraded by phagocytosis and tissue fluids invasion, the fiber loss was significantly higher compared with increasing coating bilayers, and the $(\mathrm{CS} / \mathrm{COL})_{20}$ showed higher in vivo degradation ratio than other matrices. This can be attributed to the combination of better interconnected pores, higher specific surface area of electrospun nanofibers and the biodegradable CS and collagen immobilized on (PCL/collagen) ${ }_{n}$ films coatings.

For the collagen deposition and angiogenesis, the groups showed light blue fibers deposition while the control group was the least obvious one (Fig. S4A $\dagger$ ). With the addition of CS and collagen, the degree of collagen production was significantly elevated (Fig. 4SB-E $\dagger$ ). There was no obvious positive vessel in the center of the wound treated with PCL-cellulose (Fig. S4A $\dagger$ ) mats and $(\mathrm{CS} / \mathrm{COL})_{5}$ nanofibrous mats (Fig. S4B $\dagger$ ), red blood cells within vessels were achieved in regenerated tissues after treatment with LBL structured $(\mathrm{CS} / \mathrm{COL})_{10}$ fibers (Fig. S4C $\dagger$ ). As vascularization continued, the ingrowth of vessels appeared in the center of the wound with the widening of small vessels, especially in $(\mathrm{CS} / \mathrm{COL})_{15}$ and $(\mathrm{CS} / \mathrm{COL})_{20}$ nanofibrous mats (Fig. S4D and E $\dagger$ ), while few blood vessels were found in the edge of wound with variable lumen sizes, which further facilitated capillary-like tube formation in vitro and integration with host vessels in vivo.

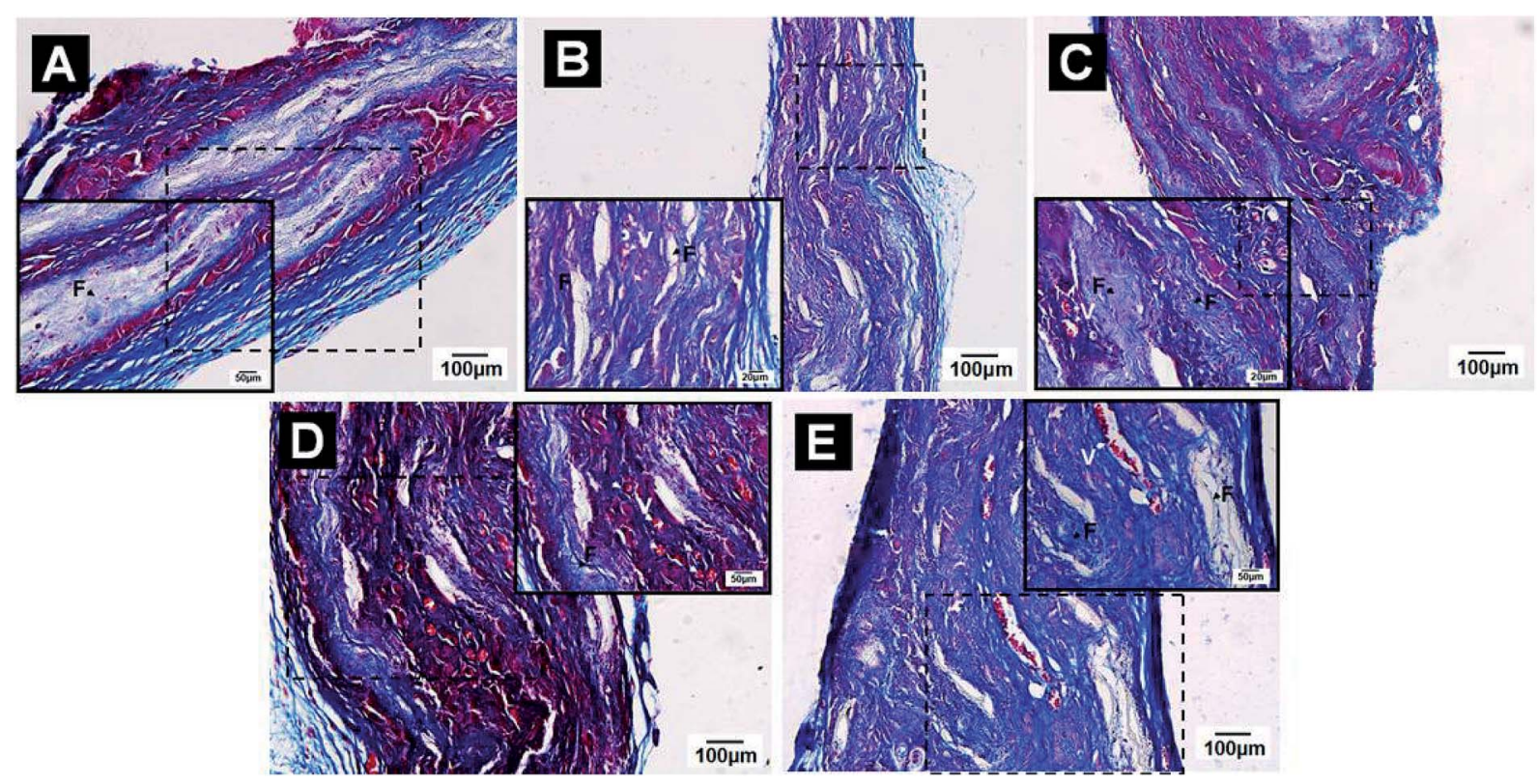

Fig. 10 Masson trichrome staining of the aligned (A) PCL-cellulose and nanofibrous mats coated by $(C S / C O L)_{n}:(B)(C S / C O L)_{5},(C)(C S / C O L)_{10}$; (D) $(\mathrm{CS} / \mathrm{COL})_{15} ;(\mathrm{E})(\mathrm{CS} / \mathrm{COL})_{20}$ together with wound areas after subcutaneous implantation for 4 weeks. The black arrows represented remaining scaffolds, and white ones represented signs of vascularization. 
At 4 weeks, this phenomenon was more obvious in (CS/ $\mathrm{COL})_{5},(\mathrm{CS} / \mathrm{COL})_{10},(\mathrm{CS} / \mathrm{COL})_{15}$ and $(\mathrm{CS} / \mathrm{COL})_{20}$ nanofibrous mats than the group without LBL modification, suggesting that the supplement of CS and collagen could enhance the COL synthesis. Besides, the neovascular structures were also visualized with larger magnification. There were few cells penetrating into the center of the scaffolds in the PCL-cellulose and (CS/ $\mathrm{COL})_{5},(\mathrm{CS} / \mathrm{COL})_{10}$ group. By comparison, more fibroblast-like cells grew into the $(\mathrm{CS} / \mathrm{COL})_{20}$ scaffolds when pre-seeded with VECs. Most cell infiltration was observed in the group and displayed the most densely filled extracellular matrix (ECM). The small blood vessels could be found in VEC-seeded LBL modified scaffold constructs that strongly encouraged the vascularization around the damaged tissues. A significant increase in vessel number and lumen area in $(\mathrm{CS} / \mathrm{COL})_{10},(\mathrm{CS} /$ $\mathrm{COL})_{15}$ and $(\mathrm{CS} / \mathrm{COL})_{20}$ nanofibrous mats groups when compared with control and $(\mathrm{CS} / \mathrm{COL})_{5}$ groups. More importantly, the collagen IV positive vessels were scarce and mostly situated on the periphery of the wounds at the beginning (Fig. 10A). Nevertheless, the percentage of vascularized area in LBL modified nanofibers significantly increased thereafter (Fig. 10B-E). Collagen IV positive vessels were more abundant and presented wider lumen diameter relative to other conditions at week 2. A large number of microvessels was observed in $(\mathrm{CS} / \mathrm{COL})_{20}$ group, and the microvessel density was higher than those of other groups (Fig. 10E). The staining in (CS/COL $)_{20}$ films coated nanofibers for 2 and 4 weeks demonstrated that functional tubular structures were observed, suggesting that the combination of nanostructure and prevascularization with seeded VECs could support a more mature vasculature.

When implanting the scaffolds without prevascularization in vivo, although the host tissues could migrate into the scaffold, the process of radiating outwards into the scaffold was relatively slow. ${ }^{52}$ The seeded cells are prone to necrosis without a timely supply of nutrients. Our present study combined the effect of the nanofibrous microenvironment with pre-vascularization on promoting rapid vascularization of the entire large scaffold. As discussed above, the prepared porous nanofibrous scaffolds not only facilitate cell infiltration and distribution during cell seeding, but also enhance the delivery of oxygen and nutrients into the scaffold bulk. During in vitro pre-vascularization, endothelial cells were widely distributed throughout the scaffold and formed capillary-like tubes, which subsequently integrated with the rapidly infiltrated host vessels after implantation in vivo. On the whole, the prepared nanofibrous mats and pre-seeded HUVECs synergistically promoted rapid vascularization through the entire large porous scaffolds, which provided a promising strategy for developing implantable functional vascular grafts by promoting rapid vascularization and enhancing the survival of seeded cells.

\section{Experimental}

\subsection{Materials}

Poly( $\varepsilon$-caprolactone) (PCL, $M_{\mathrm{w}}=70-90 \mathrm{kDa}$ ), cellulose acetate $\left(\mathrm{CA}, M_{\mathrm{n}}=3 \times 10^{4} \mathrm{Da}\right)$ and type I collagen (from calf skin) were purchased from Sigma Aldrich Co., USA. Chitosan (CS, $M_{\mathrm{w}}=2.1$ $\times 10^{5} \mathrm{Da}, \mathrm{DD}=92 \%$ ) was provided by Yuhuan Ocean Biochemical Co. (Taizhou, China). All other chemicals were of analytical grade and used as received. Purified water was prepared by a system consisting of three units (active charcoal, ion exchanger, and reverse osmosis) connected in series to an ELGA water purification system (PURELAB ultra, UK). All aqueous solutions were prepared with purified water (electrical resistivity $=18.2 \mathrm{M} \Omega \mathrm{cm}$ ).

\subsection{Preparation and modification of nanofibrous matrices}

$8 \mathrm{wt} \%$ PCL solutions and $14 \mathrm{wt} \%$ CA solutions were prepared separately by dissolving PCL in a mixture of dichloromethane (DCM) and $N, N$-dimethylformamide (DMF) with a volume ratio of $2: 1$ and CA in acetone/DMAC $(2: 1, \mathrm{v} / \mathrm{v})$. The PCL and CA solutions were then mixed to obtain mixtures with weight ratios of $3: 2$, and the resultant mixtures were stirred for $3 \mathrm{~h}$. The electrospinning system applied in this work was similar to that reported previously. Briefly, a DC voltage of $16 \mathrm{kV}$ with low current output was applied between the syringe tip and a cylindrical collector. The typical distance between the syringe tip and the grounded collector was $15 \mathrm{~cm}$. Polymer solution inside the syringe was charged with a positive voltage by dipping a platinum wire into the solution from a positive lead; the plane and cylindrical collector were grounded, respectively. Isotropic (fibers collected randomly with no alignment) and anisotropic (fibers collected with alignment) PCL-cellulose fiber were obtained by hydrolysis of the mats in a $0.05 \mathrm{M} \mathrm{NaOH}$ aqueous solution at room temperature for $7 \mathrm{~d}$. The prepared mats were rinsed and thoroughly washed with purified water for three times to remove retained $\mathrm{NaOH}$, they were then vacuum-dried at ambient temperature for $48 \mathrm{~h}$. Then the LBL coating process was conducted according to our previous work. ${ }^{29}$

\subsection{Characterization of electrospun PCL/collagen nanofiber meshes}

Cold type Field Emission Scanning Electron Microscopy (FESEM) and atomic force microscopy (AFM) were used to characterize these nanofibrous matrices. For SEM examination, the dehydrated samples were sputter-coated with gold and then examined with a FE-SEM (JSM-6700F, JEOL, Japan). To determine the diameter of nanofibers, images of five randomly selected areas were captured and analyzed by Photoshop 7.0 edition. The quantitative analysis of the orientation of electrospun nanofibers was determined by using the ImageJ program along with the Oval Profile Plot plugin. The final results were presented by plotting this arbitrary scale versus degrees, where the height of peaks represents a greater degree of alignment at a given angle. ${ }^{53}$

The surface roughness of different nanofibrous matrices was determined by using Nanoscope IV atomic force microscope (Innova, Bruker AXS., USA) in tapping mode and expressed as height and phase images. Three randomly selected areas of the surface with the size of $10 \times 10 \mathrm{~mm}(x, y$ direction) were scanned, respectively. To describe the topography and roughness of the substrates, the roughness parameter for the surface, Rms 
( $R_{\mathrm{q}}$, the root-mean-square height of the surface) was calculated by Nanoscope analysis.

\subsection{Measurement of platelet adhesion on prepared nanofibers}

The protocol used to evaluate platelet adhesion on prepared isotropic nanofibrous matrices was derived from a previous report. ${ }^{54}$ Whole blood from a healthy volunteer was collected into BD Vacutainer ${ }^{\circledR}$ EDTA K2E tubes and then mixed with quinacrine dihydrochloride to label platelets. The experimental protocol was approved by the Ethics Committee for Animal Experimentation of the Fourth Military Medical University (TDLL-2015213, July 2015), collagen I-coated stainless steel surface served as positive control. $2.5 \mathrm{mg} \mathrm{mL} \mathrm{m}^{-1}$ collagen I prepared in $3 \%$ glacial acetic acid was coated on stainless steel surface. Blood samples were incubated on each surface for $2 \mathrm{~h}$ at $37{ }^{\circ} \mathrm{C}$. Platelet attachment was quantified by acquiring 5 random images on each surface at $10 \times$ magnification from three separate samples by using a CLSM. Average numbers of adhered platelets were used to evaluate the relative attachment of platelets onto the surfaces. A probability value $(p)$ of less than $0.05\left({ }^{*} p<0.05\right)$ was considered to be statistically significant.

\subsection{Cell culture and viability assay}

The human umbilical vein endothelial cells (HUVECs) were maintained in endothelial basal medium-2 (EBM-2) supplemented with $10 \%$ fetal bovine serum, 100 units per $\mathrm{mL}$ penicillin/streptomycin, $30 \mu \mathrm{g} \mathrm{mL}^{-1}$ endothelial cell growth supplement and $25 \mathrm{U} \mathrm{mL}^{-1}$ heparin sodium. The cells were maintained at a humidified atmosphere of $95 \%$ air and $5 \% \mathrm{CO}_{2}$ at $37{ }^{\circ} \mathrm{C}$. The culture medium was replaced every 3 days until cells were confluent.

3.5.1. MTT assay. The MTT assay was used to assess cell metabolic activity for the evaluation of survival and growth characteristics of HUVECs within the prepared aligned nanofibrous scaffolds. Before cell seeding, the LBL structured (CS/ $\mathrm{COL})_{n}$ aligned and randomly oriented electrospun scaffolds were cut into a determined size and sterilized by ethylene oxide gas, and washed with PBS three times and incubated with medium in DMEM medium at $37^{\circ} \mathrm{C}$. They were then placed in the refrigerator for $24 \mathrm{~h}$, after the incubation period the socalled extracts were obtained and degermed by $0.22 \mu \mathrm{m}$ filter prior to the following experiments. A total of $1 \times 10^{4}$ HUVECs were seeded in 96-well microtiter plates, after 1 day of culture in EBM, the culture medium were removed and replaced with the extraction medium mentioned above and incubated for $24 \mathrm{~h}$, and $120 \mathrm{~h}$, respectively. After that, the cells were washed gently with phosphate buffered saline (PBS) for three times, $25 \mu \mathrm{L}$ MTT (Sigma, St Louis, MO, USA) was added into targeted wells at $37{ }^{\circ} \mathrm{C}$ for $4 \mathrm{~h}$, to form formazan. Formazan crystals were dissolved in dimethyl sulfoxide (DMSO) and measured at $490 \mathrm{~nm}$ using an enzyme linked immunosorbent assay (ELISA) Reader (MODEL550, Bio-Rad, USA). All experiments were done in six replicates $(n=6)$.

3.5.2. Cell tracker labelling of HUVECs. Prior to seeding cells on scaffolds, Cell Tracker ${ }^{\mathrm{TM}}$ Red (Invitrogen Ltd, USA) were used to label HUVECs in vitro. ${ }^{55}$ Adherent cells in culture flasks were rinsed with serum free culture media before CellTracker ${ }^{\mathrm{TM}}$ Red was added to culture flasks and incubated at $37{ }^{\circ} \mathrm{C}$ for 45 minutes. After washing with Greens medium, cells were incubated in Greens medium for a further 30 minutes at $37^{\circ} \mathrm{C}$. Cells were then seeding onto scaffolds as previously described. After seeding for $1 \mathrm{~d}$ and $5 \mathrm{~d}$, labelled cells were imaged using confocal laser scanning microscope (CLSM, 510 META, Zeiss, Germany) at ex-570 nm/em-620 nm.

\subsection{Wound healing in vitro}

In vitro CytoSelect $^{\mathrm{TM}}$ 24-Well Wound Healing Assay Kit purchased from Cell Biolabs Inc. (San Diego, CA) was used to evaluate the migratory behavior of HUVECs co-cultured with isotropic and anisotropic nanofibers. Briefly, $1 \times 10^{5}$ cells suspended in culture media were seeded onto the surfaces of aligned and randomly oriented nanofibrous matrices with inserts in the middle. After $24 \mathrm{~h}$, the insert was removed to generate a consistent wound gap $(0.9 \mathrm{~mm})$ in the middle. Cells were allowed to migrate into the wound gap on various nanofibrous matrices for $0 \mathrm{~d}$ and $3 \mathrm{~d}$ and $5 \mathrm{~d}$, respectively. After fixation, cells were stained using the staining kit and images of the wound gap were taken to analyze the migration distance of target cells. At least 10 representative points along the "wound" of each sample were used for evaluating the migration rate from three separate samples for each time point. ${ }^{53}$

\subsection{Scanning electron microscopy (SEM) of cell-seeded scaffold}

The prepared mats were treated as described above, then a total of $200 \mu \mathrm{L}$ of HUVECs suspension $\left(1 \times 10^{4}\right.$ cells per $\left.\mathrm{mL}\right)$ was seeded onto the pre-soaked nanofibrous mats and placed in 24 well culture plate. The cell seeded mats were incubated at $37^{\circ} \mathrm{C}$ in a humidified atmosphere for $4 \mathrm{~h}$ to make cells diffuse into and adhere to the scaffold before the addition of culture medium into each well. Cellular constructs were harvested after 3 d. Subsequently, the cell-seeded scaffolds were replenished with fresh media every $3 \mathrm{~d}$. HUVECs-seeded mats were washed by phosphate buffer saline (PBS) twice and then fixed with $4 \%$ glutaraldehyde for $2 \mathrm{~h}$ at $4{ }^{\circ} \mathrm{C}$. The samples were then dehydrated through a series of graded ethanol solutions and freezedried. Dry constructs were sputter-coated with gold and observes by Scanning Electron Microscope (SEM) (JSM-6700F, JEOL, Japan).

\subsection{Cytoskeleton organization and phenotype study}

The cytoskeleton organization of HUVECs grown on isotropic and anisotropic nanofibers was analyzed by using actin staining. After $48 \mathrm{~h}$ cultivation, cells were fixed with $3.7 \%$ formaldehyde for $10 \mathrm{~min}$, permeabilized with $0.1 \%$ Triton $\mathrm{X}-100$ for $5 \mathrm{~min}$, stained with rhodamine-phalloidin (Sigma-Aldrich, $1: 500$ ) for $20 \mathrm{~min}$, and stained with DAPI (Sigma-Aldrich, $1: 1000$ ) for $10 \mathrm{~min}$ under dark conditions. Fibers were stained with FI-TC. The stained actin filaments and fibers were observed using LSCM. 


\subsection{Immunohistochemistry}

The expression patterns of endothelial cell marker (CD31), components of basement membrane extracellular matrix (nidogen and perlecan) and cell adhesion molecule vinculin of HUVECs were analyzed by immunocytochemistry. For immunocytochemical analysis, the cell-scaffold constructs were gently washed with PBS and were fixed with $4 \%$ paraformaldehyde for 15 min, made permeable with $0.1 \%$ Triton $\mathrm{X}$ 100 for $10 \mathrm{~min}$, blocked with 1\% bovine serum albumin (BSA) for $30 \mathrm{~min}$ at room temperature after $48 \mathrm{~h}$ cultivation, treated with the following primary antibodies, respectively: anti-CD31 (Abcom, ab119339, $1: 500$ ), anti-nidogen (sc-33141, Santa Cruz Biotechnology, Inc, $1: 500$ ), anti-perlecan (sc-25848, Santa Cruz Biotechnology, Inc, $1: 400$ ) and anti-vinculin (Abcom, ab18058, $1: 200$ ) antibody at $4{ }^{\circ} \mathrm{C}$ overnight and washed with PBS, followed by incubation with goat anti-rabbit IgG-Cy3 conjugate secondary antibody (Abcom, America) for $30 \mathrm{~min}$ at room temperature in the dark, cell nuclei were stained with DAPI (Sigma, 1 : 1000). Subsequently, the samples were washed with PBS, mounted with anti-fade mounting medium (Vector Laboratories Inc., CA, USA), sealed and stored in the dark before taking fluorescent images using confocal laser scanning microscope (CLSM, 510 META, Zeiss, Germany). Fluorescence intensity of stained sections was quantified by using an Image Pro Plus software.

\subsection{In vivo implantation}

The in vivo degradation of the electrospun scaffolds and their effect on vascular regeneration was conducted by subcutaneous implantation in SD rats. Masson trichrome staining was used to further determine the proliferation of new collagen fibers in the cell-scaffold construct implanted region, with particular attention to the structural characteristics together with evidence of cellular infiltrates and angiogenesis. Briefly, male SpragueDawley rats $(250 \pm 10 \mathrm{~g})$ were used in this study. The experimental protocol was approved by the Ethics Committee for Animal Experimentation of the Fourth Military Medical University (TDLL-2015213, July 2015), which also met the Guide for the Care and Use of Laboratory Animals of the National Institutes of Health. All surgery was performed under anesthesia, and all efforts were made to minimize suffering. After anesthetization with $1 \%$ pentobarbital sodium for $5 \mathrm{~min}$, the hairs on the backs of the rats were shaved, the skin was dissected over about $30 \mathrm{~mm}$ at two sites parallel with the vertebral column and the outer membrane under the skin was stripped. The scaffolds (diameter $=20 \mathrm{~mm}$ ) and HUVECsseeded aligned nanofibrous mats were implanted in the SD rats after dissection and sutured under aseptic condition. The experiment was repeated for three times. After surgery, the animals were given antibiotics twice daily for $48 \mathrm{~h}$. The rats were sacrificed to retrieve the residual scaffolds for analysis after implantation for 14 and 28 days, respectively. Three rats were used for each data point. The harvested mats were washed in PBS, fixed in $10 \%$ neutral buffered formalin, dehydrated through a series of graded alcohols, embedded in paraffin and sectioned at a thickness of $5 \mu \mathrm{m}$. Sections were then deparaffinized and Masson trichrome staining was performed, all the sections were observed under light microscope (Nikon Eclipse E400, Japan) for analysis.

\subsection{Statistical analysis}

Unless otherwise indicated, all the quantitative results were expressed as means \pm standard deviation (SD). Whenever appropriate, two-tailed Student's $t$-test was used to discern the statistical difference between groups. A probability value $(p)$ of less than $0.05(* p<0.05, * * p<0.01)$ was considered to be statistically significant.

\section{Conclusion}

Collectively, a new strategy was developed for tissue regeneration by combining LBL modified nanofibrous mats with prevascularization with VECs to promote rapid vascularization and integration in vivo. We successfully fabricated nanofibrous PCL-cellulose scaffolds coated by bioactive chitosan and collagen that mimicked the ECM to promote HUVECs infiltration, survival, proliferation, migration and capillary-like tube formation during in vitro prevascularization, the nanofiber meshes fabricated by electrospinning showed a unidirectional fiber orientation that could guide HUVECs alignment and enhance capillary-like tube formation. CS/COL-coated nanofibers allowed HUVEC cells to adhere well and spread with high viability. A cytoskeleton was developed with well-stretched actin bundles, and endothelial cell markers, such as CD31, nidogen, perlecan and vinculin were positively expressed in the presence of the aligned nanofibrous mats, following transplantation in vivo, these matrices also promoted host vessel infiltration deep into the scaffolds and integration with in vitro prefabricated vascular structures. Hence the prepared nanofibrous scaffold and pre-seeded HUVECs promoted rapid vascularization of scaffolds. Taken together, this study indicated that the LBL modification on electrospun nanofibers is a promising and effective strategy for vascular tissue engineering that requires efficient endothelialization of graft surfaces.

\section{Acknowledgements}

This work was funded by National High Technology Research and Development Program of China (863 Program) (Grant No. SS2015AA020313), the National Natural Science Foundation of China (Grant No. 81272134, 81401597), Science and Technology Innovation Development Foundation of Tangdu hospital (Grant No. 2013CXTS016) and the Science and Technology Coordinating Innovative Engineering Project of Shaanxi Province (2014KTCQ03-01). The authors thank Zhende medical Co. Ltd for their technical support.

\section{References}

1 M. J. B. Wissink, R. Beernink, A. A. Poot, G. H. M. Engbers, T. Beugeling, W. G. V. Aken and J. Feijen, J. Controlled Release, 2000, 64, 103-114. 
2 X. Wang, P. Lin, Q. Yao and C. Chen, World J. Surg., 2007, 31, 682-689.

3 H. P. Greisler, S. Johnson, K. Joyce, S. Henderson, N. M. Patel, T. Alkhamis, R. Beissinger and D. U. Kim, Arch. Surg., 1990, 125, 1622-1625.

4 P. Zilla, P. Preiss, P. Groscurth, F. Rösemeier, M. Deutsch, J. Odell, C. Heidinger, R. Fasol and O. U. Von, Surgery, 1994, 116, 524-534.

5 H. Liu, X. Li, G. Zhou, H. Fan and Y. Fan, Biomaterials, 2011, 32, 3784-3793.

6 M. Lovett, K. Lee, A. Edwards and D. L. Kaplan, Tissue Eng., Part B, 2009, 15, 353-370.

7 R. E. Unger, A. Sartoris, K. Peters, A. Motta, C. Migliaresi, M. Kunkel, U. Bulnheim, J. Rychly and C. J. Kirkpatrick, Biomaterials, 2007, 28, 3965-3976.

8 D. L. Hutton and W. L. Grayson, Curr. Opin. Chem. Eng., 2014, 3, 75-82.

9 A. Bader, G. Steinhoff, K. Strobl, T. Schilling, G. Brandes, H. Mertsching, D. Tsikas, J. Froelich and A. Haverich, Transplantation, 2000, 70, 7-14.

10 N. L. James, K. Schindhelm, P. Slowiaczek, B. K. Milthorpe, N. P. B. Dudman, G. Johnson and J. G. Steele, Artif. Organs, 1990, 14, 355-360.

11 S. Kaushal, G. E. Amiel, K. J. Guleserian, O. M. Shapira, T. Perry, F. W. Sutherland, E. Rabkin, A. M. Moran, F. J. Schoen, A. Atala, S. Soker, J. Bischoff and J. E. Mayer Jr, Nat. Med., 2001, 7, 1035-1040.

12 B. W. Tillman, S. K. Yazdani, J. L. Sang, R. L. Geary, A. Atala and J. J. Yoo, Biomaterials, 2009, 30, 583-588.

13 S. H. Ku and B. P. Chan, Biomaterials, 2010, 31, 9431-9437.

14 M. P. Lutolf, P. M. Gilbert and H. M. Blau, Nature, 2011, 462, 433-441.

15 M. P. Lutolf and J. A. Hubbell, Nat. Biotechnol., 2005, 23, 4755.

16 W. Zheng, Z. Wang, L. Song, Q. Zhao, J. Zhang, D. Li, S. Wang, J. Han, X. L. Zheng and Z. Yang, Biomaterials, 2012, 33, 2880-2891.

17 H. H. Sun, T. J. Qu, X. H. Zhang, Q. Yu and F. M. Chen, Biotechnol. Prog., 2012, 28, 3-20.

18 M. Matsui and Y. Tabata, Acta Biomater., 2012, 8, 1792-1801.

19 E. S. Place, N. D. Evans and M. M. Stevens, Nat. Mater., 2009, 8, 457-470.

20 F. M. Chen, M. Zhang and Z. F. Wu, Biomaterials, 2010, 31, 6279-6308.

21 X. Hao, E. A. Silva, A. Månsson-Broberg, K. H. Grinnemo, A. J. Siddiqui, G. Dellgren, E. Wärdell, L. A. Brodin, D. J. Mooney and C. Sylvén, Cardiovasc. Res., 2007, 75, 178185.

22 S. Sahoo, L. T. Ang, C. H. Goh and S. L. Toh, J. Biomed. Mater. Res., Part A, 2010, 93, 1539-1550.

23 Y. Yoshikawa and S. O. Abrahamsson, Acta Orthop. Scand., 2001, 72, 287-292.

24 Y. Zhang, J. R. Venugopal, A. El-Turki, S. Ramakrishna, B. Su and C. T. Lim, Biomaterials, 2008, 29, 4314-4322.

25 J. Han, P. Lazarovici, C. Pomerantz, X. Chen, Y. Wei and P. I. Lelkes, Biomacromolecules, 2011, 12, 399-408.
26 N. F. Huang, J. Okogbaa, J. C. Lee, A. Jha, T. S. Zaitseva, M. V. Paukshto, J. S. Sun, N. Punjya, G. G. Fuller and J. P. Cooke, Biomaterials, 2013, 34, 4038-4047.

27 E. S. Lai, N. F. Huang, J. P. Cooke and G. G. Fuller, Regener. Med., 2012, 7, 649-661.

28 K. H. Nakayama, G. Hong, J. C. Lee, J. Patel, B. Edwards, T. S. Zaitseva, M. V. Paukshto, H. Dai, J. P. Cooke and Y. J. Woo, ACS Nano, 2015, 9, 6900.

29 H. Rong, W. Li, X. Lv, Z. Lei, Y. Bian, H. Deng, H. Wang, J. Li and X. Li, Biomaterials, 2015, 53, 58-75.

30 G. Decher, Science, 1997, 277, 1232-1237.

31 Q. He, K. Gong, Q. Ao, T. Ma, Y. Yan, Y. Gong and X. Zhang, J. Biomater. Appl., 2013, 27, 1032-1045.

32 J. M. Corey, D. Y. Lin, K. B. Mycek, Q. Chen, S. Samuel, E. L. Feldman and D. C. Martin, J. Biomed. Mater. Res., Part A, 2007, 83, 636-645.

33 R. Gruschwitz, J. Friedrichs, M. Valtink, C. M. Franz, D. J. Müller, R. H. Funk and K. Engelmann, Invest. Ophthalmol. Visual Sci., 2010, 51, 6303-6310.

34 W. Zhang, L. S. Wray, J. Rnjak-Kovacina, X. Ling, D. Zou, S. Wang, M. Zhang, J. Dong, G. Li and D. L. Kaplan, Biomaterials, 2015, 56, 68-77.

35 U. Kneser, L. Stangenberg, J. Ohnolz, O. Buettner, J. SternStraeter, D. Möbest, R. E. Horch, G. B. Stark and D. J. Schaefer, J. Cell. Mol. Med., 2006, 10, 695-707.

36 L. Bacakova, E. Filova, M. Parizek, T. Ruml and V. Svorcik, Biotechnol. Adv., 2011, 29, 739-767.

37 P. Solař, O. Kylián, A. Marek, M. Vandrovcová, L. Bačáková, J. Hanuš, J. Vyskočil, D. Slavínská and H. Biederman, Appl. Surf. Sci., 2014, 324, 99-105.

38 C. Xu, F. Yang, S. Wang and S. Ramakrishna, J. Biomed. Mater. Res., Part A, 2004, 71, 154-161.

39 V. Milleret, T. Hefti, H. Hall, V. Vogel and D. Eberli, Acta Biomater., 2012, 8, 4349-4356.

40 J. Lee, Y. Ju, W. Lee, K. Park and Y. Kim, J. Biomed. Mater. Res., 1998, 40, 314-323.

41 D. A. Rubenstein, S. M. Venkitachalam, D. Zamfir, F. Wang, H. Lu, M. D. Frame and W. Yin, J. Biomater. Sci., Polym. Ed., 2010, 21, 1713-1736.

42 B. Dhandayuthapani, S. H. Varghese, R. G. Aswathy, Y. Yoshida, T. Maekawa and D. Sakthikumar, Int. J. Biomater., 2012, 2012, 345029.

43 E. C. Novosel, C. Kleinhans and P. J. Kluger, Adv. Drug Delivery Rev., 2011, 63, 300-311.

44 Z. Lokmic and G. M. Mitchell, Tissue Eng., Part B, 2008, 14, 87-103.

45 Y. H. Nien, J. Y. Wang and Y. S. Tsai, J. Nanosci. Nanotechnol., 2013, 13, 4703-4707.

46 P. Baluk, S. Morikawa, A. Haskell, M. Mancuso and D. M. Mcdonald, Am. J. Pathol., 2003, 163, 1801-1815.

47 E. Kohfeldt, T. Sasaki, W. Göhring and R. Timpl, J. Mol. Biol., 1998, 282, 99-109.

48 M. Hopf, W. Göhring, A. Ries, R. Timpl and E. Hohenester, Nat. Struct. Biol., 2001, 8, 634-640.

49 M. Handler, P. D. Yurchenco and R. V. Iozzo, Dev. Dyn., 1997, 210, 130-145. 
50 T. Escalante, N. Ortiz, A. Rucavado, E. F. Sanchez, M. Richardson, J. W. Fox and J. M. Gutiérrez, PLoS One, 2011, 6, e28017.

51 E. Gustafsson, M. Almonte-Becerril, W. Bloch and M. Costell, PLoS One, 2013, 8, 252-262.

52 J. Rnjak-Kovacina, L. S. Wray, J. M. Golinski and D. L. Kaplan, Adv. Funct. Mater., 2014, 24, 2188-2196.
53 C. Huang, X. Fu, J. Liu, Y. Qi, S. Li and H. Wang, The involvement of integrin $\beta 1$ signaling in the migration and myofibroblastic differentiation of skin fibroblasts on anisotropic collagen-containing nanofibers, Elsevier, AP, 2014. 54 H. Ceylan, A. B. Tekinay and M. O. Guler, Biomaterials, 2011, 32, 8797-8805.

55 F. J. Bye, A. J. Bullock, R. Singh, F. Sefat, S. Roman and S. Macneil, J. Biomater. Tissue Eng., 2014, 4, 686-692. 